\title{
Some Ethnomedicinal Plants of Western Himalayas Useful in Making Local Alcoholic Drinks
}

\author{
Tara Devi Sen ${ }^{1}$, Tanuja Thakur ${ }^{2}$ \\ ${ }^{I}$ Assistant Professor. Department of Botany, Sardar Vallabhbhai Patel. Cluster University, Mandi, \\ India \\ ${ }^{2}$ Research Associate. Tanuja Thakur Vill.Kusmal, P.O. Saletar, Teh. Kotli, Distt. Mandi,India
}

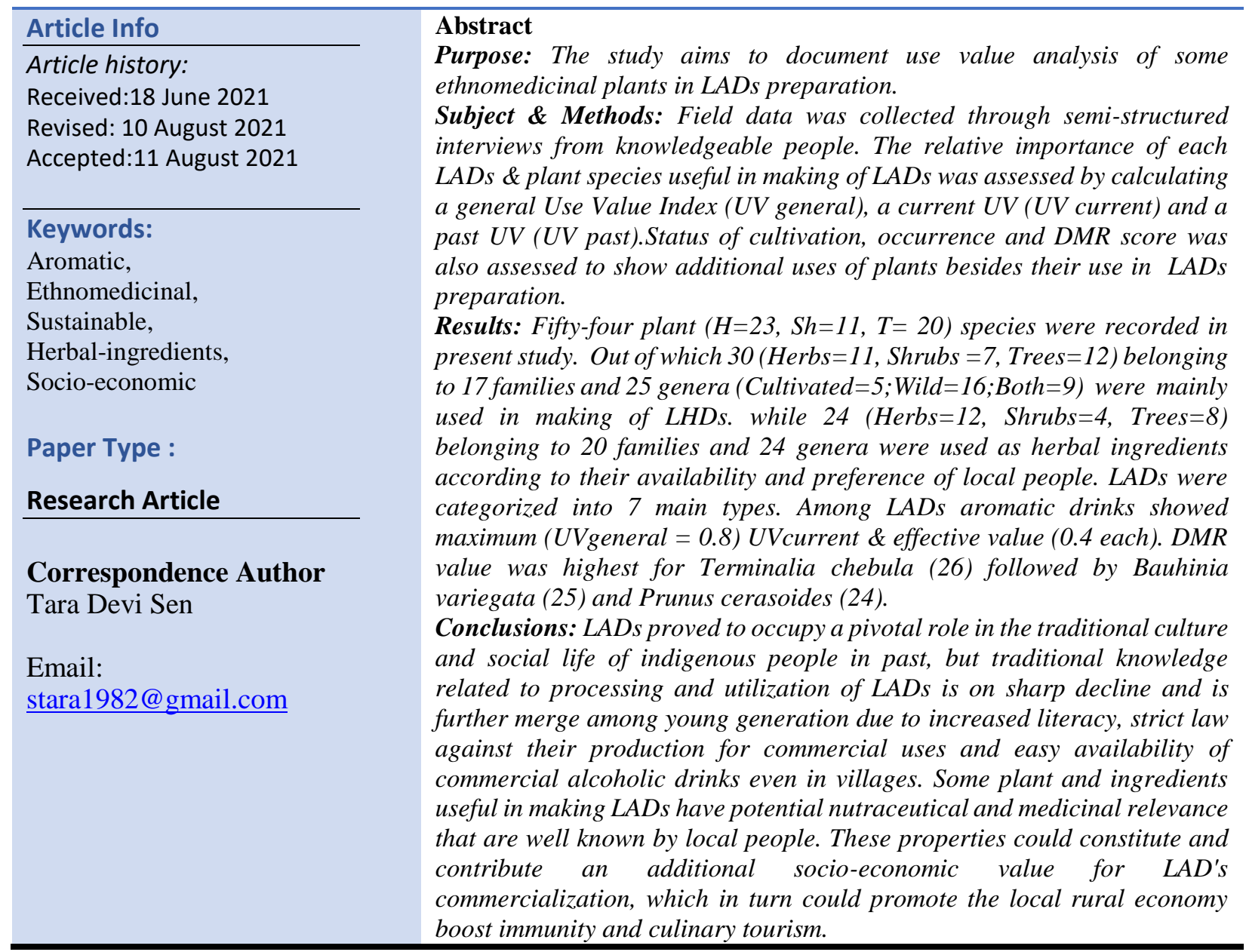

\section{Introduction}

Local alcoholic drinks (LADs) are homemade and informal preparations produced at local or family level for their nutraceutical, medicinal and socio-economic values. The World Health Organization (WHO, 2014) includes these alcoholic drinks in the so-called "unrecorded alcohol," highlighting their cultural, social and economic importance around the world. It has been estimated that almost one-quarter $(24.8 \%)$ of all the alcohol consumed worldwide is drunk in the form of unrecorded alcohol (WHO, 2014). In some countries, this unrecorded alcohol consumption goes up more than $50 \%$ of total alcohol consumption (WHO, 2014).The preparation and consumption of many LADs emerged mainly due to the climatic conditions 
and discovering the use and importance of surrounding natural resources (Roy et al., 2004). A wide range of LADs can be found in different corners of the globe and quite often the social context in which these drinks are produced and consumed is also of great interest (WHO, 2014). LADs have been a distinctive component of many cultures of the world including India for thousands of years (Egea et al.,2016, Thakur, 2021). Indian most valued religious scripts like "Ramayana" also mentions the consumption of alcohol millions of years ago by "Asuras" (Abhijit Das. 2016). LADs are still an important part of the traditional knowledge in many human communities (Śliwińska et al., 2017) and plays a significant role in the socio- economic and cultural life of the tribal or rural people.

Consumption of LADs has been found to be associated with many occasions, social gatherings and ritual like festivals, marriages birth or even death ceremonies (Saikia et al., 2007) in many communities around the globe, traces of these traditional customs originating from village societies and tribes still persist and also being utilized for medical purposes too (Tardío et al.,2006; Lejeune et al.,2003; Madej ,Pirożnikow et al.,2014; Austin,1985; Deka \& Sarma 2010). For example it is used to be a common practice to take LADs by woman after giving birth in villages and even still practiced in remote areas. In those human communities where alcohol was traditionally consumed, production of LADs commonly occurred on a small scale as a household or artisanal activity, particularly where or when the agricultural surpluses were available (WHO, 2014). In past local community is largely involved in production and commercial selling of such type of liquors but with strict law against production for commercial uses, this practice is limited to very few communities specially those residing in temperate hilly areas where it is consumed to keep body warm in extreme winters. People of these areas prepare LADs for their local personal use or sometime sell it illegely to nearby villages (Thakur, 2021). They also add many herbal ingredients, which are well known for their rich medicinal and nutraceutical values to these drinks for better health and to boost immunity, but with the passage of time, this practice of making homemade LADs is losing ground and need to be revitalize (Sen, 2021).

Some destination of study area were famous for production and selling of aromatic and medicinal drinks. These places are favorite tourist destination for the tourist of culinary tourism industry and source of income for locals involved illegely in production and selling of LADs. Further with advancement in society, increased literacy rate and urbanization there is sharp decline in indigenous knowledge related to processing of LADs. Also LADs have only received marginal attention from researchers and ethnobotanists round the world. As a result focused studies on production and consumption pattern of LADs has not been attempted so far specially in Mandi area of Himachal Pradesh, North Western Himalayas.

\section{Material \& Methods}

\section{Study area}

Study was done in Mandi region (31 $42^{\prime} 29.4^{\prime \prime} \mathrm{N}$ latitudes and $76^{\circ} 55^{\prime} 52.92^{\prime \prime} \mathrm{E}$ longitudes) of Himachal Pradesh, North West Himalaya (Fig.1). It covers approximately 3,950 $\mathrm{km} 2$ area comprising 469 panchayats and 3374 villages with 2, 19, 145 households. The total human populations of study area is $9,99,777$ and livestock population is 67355.Altitudinal 
range of study area varies from 500-4034 m. It supports diverse habitats, species, communities and Ecosystems. The vegetation mainly is of sub- tropical and temperate types and mostly dominated by broad leaved deciduous and evergreen and coniferous types. (District Economic and Statistical Department, Mandi, H.P).

\section{Method}

For survey and sampling (Rapid) of plants used in making LADs 10 villages were selected at different altitudinal zone of study area lying between alms 500-4034 m. Information on altitudinal range, habit, habitat (s) and utilization pattern of plants useful in making of LADs was gathered by interviewing knowledgeable persons involved in production and consumption of LADs through a questionnaire. Identification of samples was done with the help of local and regional floras (Chowdhary \& Wadhwa, 1984: Collett,1902, Dhaliwal \& Sharma,1999; Singh, 1918). The relative importance of each type of LADs \& plant species used to prepare LADs was assessed by calculating a general Use Value Index (UVgeneral), a current UV (UVcurrent) and a past UV (UVpast) following (Rossato et al. 1999). DMR score was also assessed for 30 plants species showing their additional uses besides LADs preparation. (Table 1,2, 5 \& 6; Fig .1)

Table 1: Ten Villages included to gather information

\begin{tabular}{|l|l|l|l|l|}
\hline S.No. & Village name & Latitude & Longitude & Altitude (in m) \\
\hline 1. & Dehar & $31^{\circ} 42^{\prime} 48.1^{\prime \prime} \mathrm{N}$ & $76^{\circ} 81^{\prime} 48.6^{\prime \prime} \mathrm{E}$ & $546 \mathrm{~m}$ \\
\hline 2. & kasan & $31^{\circ} 79^{\prime} 90.1^{\prime \prime} \mathrm{N}$ & $76^{\circ} 85^{\prime} 27.6^{\prime \prime} \mathrm{E}$ & $893 \mathrm{~m}$ \\
\hline 3. & Hanogi & $31^{\circ} 69^{\prime} 15.6^{\prime \prime} \mathrm{N}$ & $77^{\circ} 12^{\prime} 96.0^{\prime \prime} \mathrm{W}$ & $953 \mathrm{~m}$ \\
\hline 4. & Kusmal & $31^{\circ} 76^{\prime} 11.2^{\prime \prime} \mathrm{N}$ & $76^{\circ} 86^{\prime} 18.2^{\prime \prime} \mathrm{E}$ & $1129 \mathrm{~m}$ \\
\hline 5. & Karsog & $31^{\circ} 34^{\prime} 05.5^{\prime \prime} \mathrm{N}$ & $77^{\circ} 19^{\prime} 94.1^{\prime \prime} \mathrm{W}$ & $1362 \mathrm{~m}$ \\
\hline 6. & Tryambal & $31^{\circ} 78^{\prime} 18.9^{\prime \prime} \mathrm{N}$ & $76^{\circ} 95^{\prime} 19.1 " \mathrm{E}$ & $1383 \mathrm{~m}$ \\
\hline 7. & Padhar & $31^{\circ} 92^{\prime} 98.3^{\prime \prime} \mathrm{N}$ & $76^{\circ} 94^{\prime} 33.3^{\prime \prime} \mathrm{E}$ & $1731 \mathrm{~m}$ \\
\hline 8. & Janjheli & $31^{\circ} 50^{\prime} 65.5^{\prime \prime} \mathrm{N}$ & $77^{\circ} 21^{\prime} 32.9^{\prime \prime} \mathrm{W}$ & $2157 \mathrm{~m}$ \\
\hline 9. & Bagachanogi & $31^{\circ} 61^{\prime} 73.4^{\prime \prime} \mathrm{N}$ & $77^{\circ} 20^{\prime} 19.0^{\prime \prime} \mathrm{W}$ & $2442 \mathrm{~m}$ \\
\hline 10. & Bhalwar & $31^{\circ} 51^{\prime} 53.6^{\prime \prime} \mathrm{N}$ & $77^{\circ} 26^{\prime} 55.7^{\prime \prime} \mathrm{W}$ & $3071 \mathrm{~m}$ \\
\hline
\end{tabular}

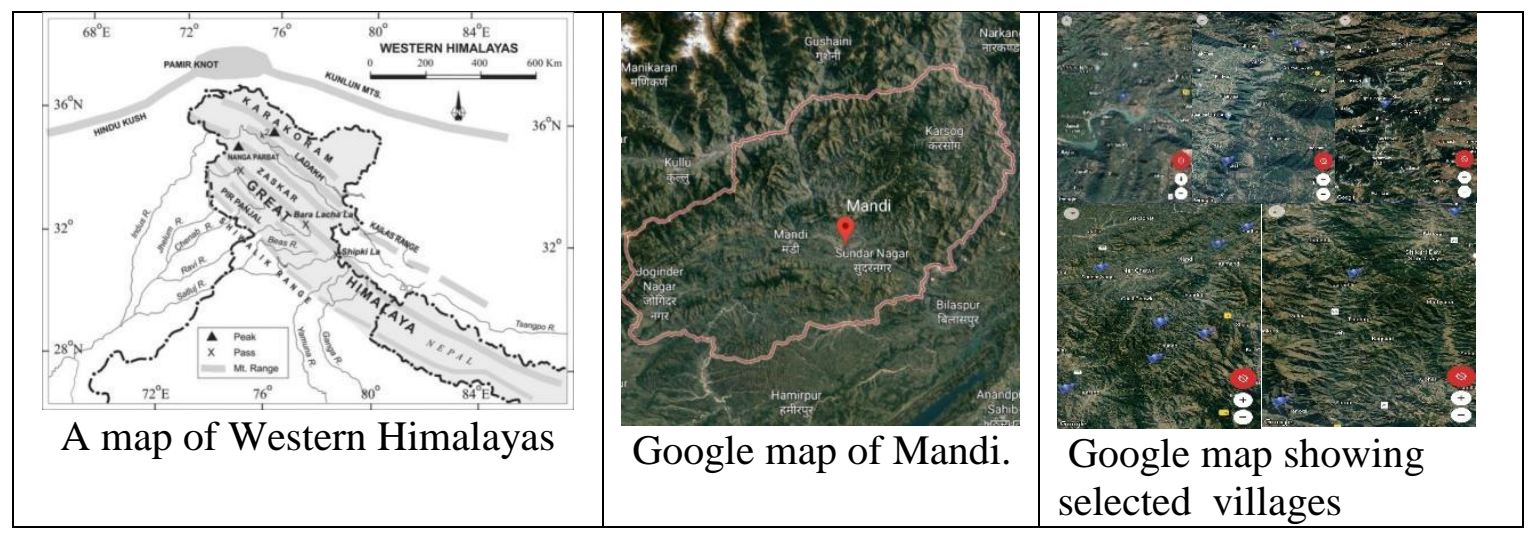

Figure 1: Map of study area

Table 2: Gender, age classes, literacy level, and occupation of people interviewed

\begin{tabular}{|l|l|l|}
\hline Gender & Density & \%Contribution \\
\hline Male & 19 & 63.33 \\
\hline Female & 11 & 36.66 \\
\hline Age classes & 2 & 6.66 \\
\hline $25-30$ & 1 & 3.33 \\
\hline $31-35$ & \multicolumn{2}{|l|}{} \\
\hline
\end{tabular}

77

Copyright (C) 2020-2021, Journal of Scientific Research in Medical and Biological Sciences (JSRMBS), Under the license CC BY- 4.0 


\begin{tabular}{|l|l|l|}
\hline $36-40$ & 2 & 6.66 \\
\hline $41-45$ & 3 & 10 \\
\hline $46-50$ & 4 & 13.33 \\
\hline $51-55$ & 6 & 20 \\
\hline $56-60$ & 4 & 13.33 \\
\hline $61-65$ & 4 & 13.33 \\
\hline $66-70$ & 3 & 10 \\
\hline $71-75$ & 1 & 3.33 \\
\hline Literacy level & \multicolumn{2}{|l|}{} \\
\hline Illiterate & 9 & 30 \\
\hline Middle & 5 & 16.66 \\
\hline Secondary & 10 & 33.33 \\
\hline Higher Seocndary & 6 & 20 \\
\hline Graduate & - & - \\
\hline Professional level & \multicolumn{2}{|l|}{} \\
\hline House wives & 8 & 20 \\
\hline Teacher & 3 & 10 \\
\hline Shopkeepers & 4 & 13.33 \\
\hline Farmers & 8 & 33.33 \\
\hline Labors & 3 & 10 \\
\hline Hakeem \& nomads & 4 & 13.33 \\
\hline
\end{tabular}

\section{Data Collection}

10 Villages of study area located in different altitude were selected and 30 informants were interviewed about preference of plant they used for making LADs, availability of useful plant parts and percentage of consumption. Data collected on LADs was organized in a simple database using Microsoft Excel. Each row (elementary record) represents a citation, defined as a single use reported for a single plant by a single informant (Signorini et.al.2009). Distinct citations based on available literature were considered for those differing from one another in at least one of the following data: species, informant and the category of use (i.e., distillates, un-distillates, aromatic, Hard -cider, Lugadi, Whiskey, Medicinal).

Citations differing in minor aspects, such as the part of the plant used, were combined into a single citation. The number of uses was obtained by considering as distinct uses for each species those differing in category of use. In the columns of the table, the following attributes are reported for each citation: as botanical names, family, local name/s, informant name, category of use, used plant part and all the other information concerning plant use collected in the interviews. Data was organized and sorted using the program EB tools (Signorini and Ongaro, unpubl.), a collection of scripts in Visual Basic for Applications in Microsoft Excel that performs advanced sorting, filtering, and counting of data based on specific user requirements.

Use Value index (UV) proposed by (Rossato et al. 1999) was used to assess the relative importance of each LADs \& plant species used in the preparation of LADs. Different types of UV was calculated : a General Use Value (UV general), based on citations of any LADs of that species recorded in the interviews; a Current Use Value (UVcurrent), based only on the citations of plants reported by the informants for uses still practiced at the present time; a Past Use Value (UVpast), based on the citations of plants reported by the informants as used only in the past. Personal experience of use was also assessed by calculating an Effective Use Value 
(UVeffective), based on citations of uses directly experienced by the informants, and a Potential Use Value (UVpotential)based on uses known, but never practiced by them.To access DMR value to know the multiple uses of plants beside their use in LADs criteria for data collection and analysis was ( $0=$ not used, $1=$ least used, $2=$ less, $3=$ good, $4=$ very good, $5=$ =best $)$.

\section{Result \& Discussion}

\section{Diversity, distribution, status and plant parts useful in making LADs}

Fifty-four plant $(\mathrm{H}=23, \mathrm{Sh}=11, \mathrm{~T}=20)$ species were recorded in present study out of which 30 (Herbs $=11$, Shrubs $=7$, Trees $=12$ ) belonging to 17 families and 25 genera were mainly used in making of LHDs. Family Rosaceae was found dominant represented by $8 \mathrm{spp}$ followed by family Poaceae 4 spp.), Rosaceae (3 spp) and Fabaceae or Araceae (represented by 2 spp. each). Most useful plant part useful in making LADS is Fr (8) followed by Rt (6) and $\mathrm{Br}$ (5) (Table.4; Fig.2 \& 4). Twenty four plant species (Herbs $=12$, Shrubs $=4$, Trees $=8$ ) belonging to 20 families and 24 genera were used as herbal ingredients or flavouring agent in LADs according to their availability and preference of local people. Family Rutaceae was found dominant represented by 4 species followed by family Rosaceae represented by 2 spp. Most important plant part used as herbal ingredient is leaf (10) followed by fruits (9) (Table.5 ; Fig. 3 \& 5). Out of 30 plant species documented for making LADs, 22 were abundantly present while 8 species were found rare and 3 of them namely Angelica glauca. Berberis lycium \& Dioscorea deltoidea were already listed in endangered IUCN Red List Category. But according to their past status they were also abundant in environment and there decrease in no. is due to fragmentation of habitat and change in climatic condition. So, these plants need sustainable harvesting, cultivation and utilization.

Among 30 plants documented for making LADs $5 \mathrm{spp}$. were found cultivated, $16 \mathrm{spp}$ were wild and 9 were both wild and cultivated (Table.4; Fig. $6 \& 7$ ).

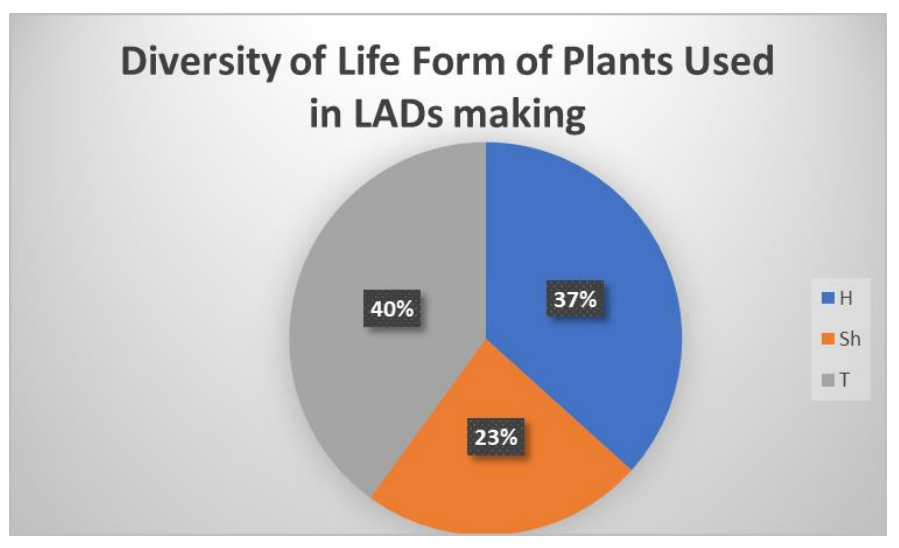

Figure 2: Diversity of plants useful in prepartion of LADs. 


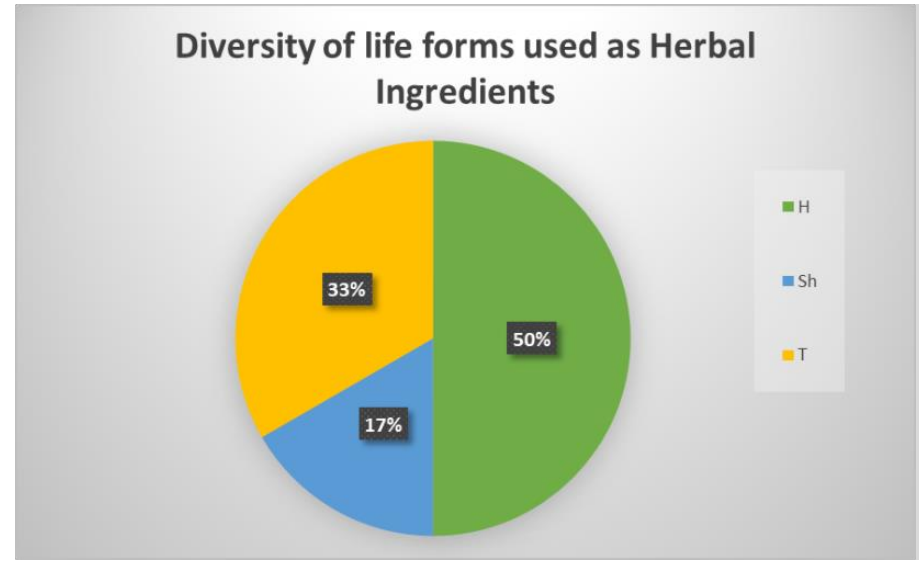

Figure 3: Diversity of plants used as herbal ingredients in LADs.

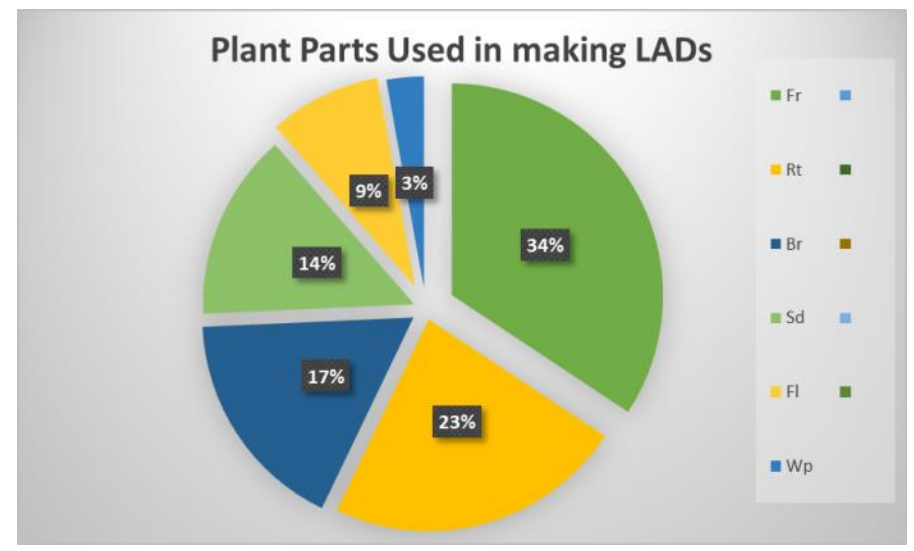

Figure 4: Diversity of plant parts useful in LADs

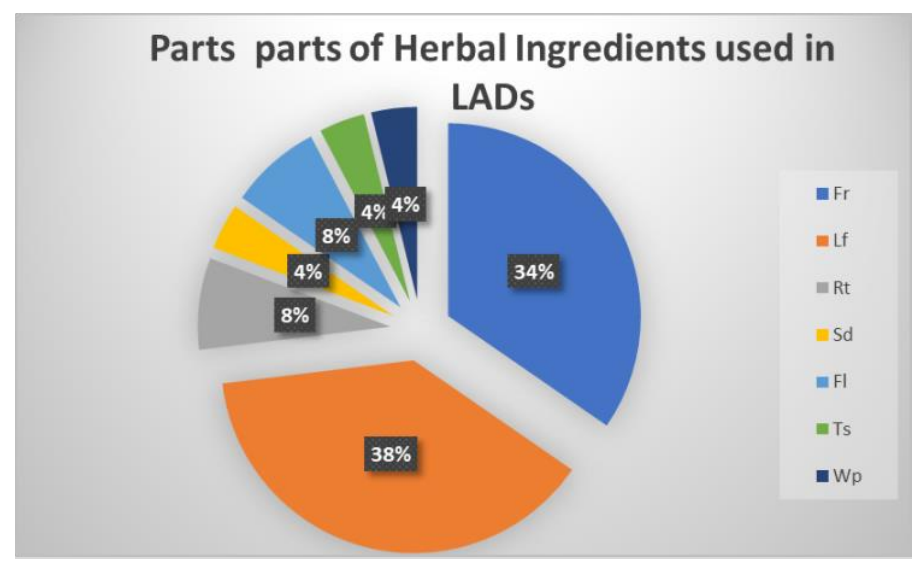

Figure 5: Diversity of plant parts of herbal ingredients useful in LADs 


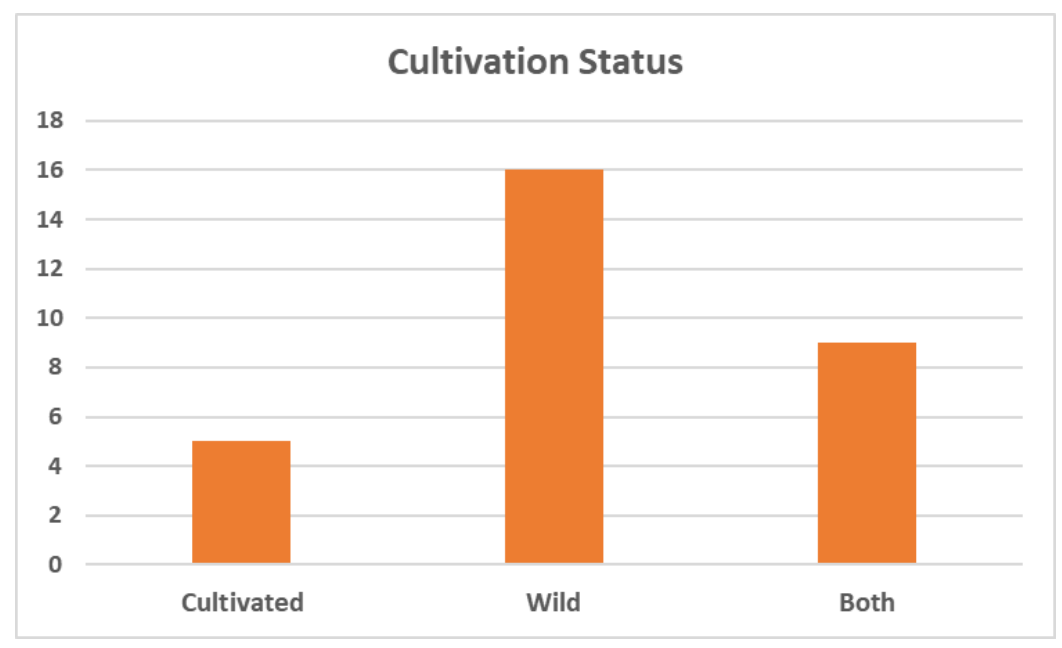

Figure 6: Cultivation status of LADs

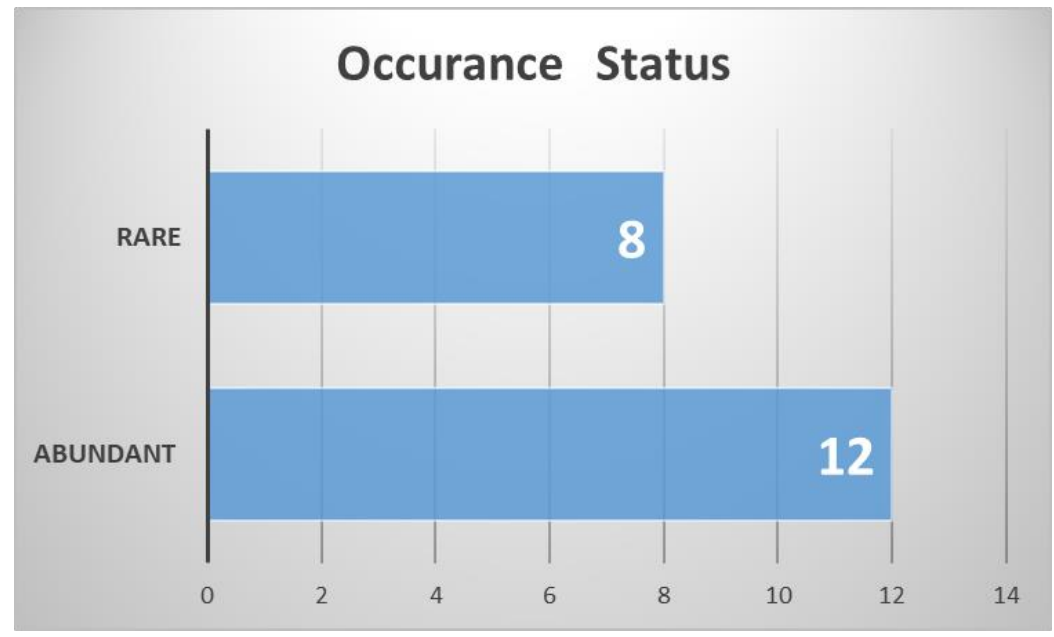

Figure 7: Occurrence Status of LADs

Table 3: Diversity, distribution, status, plant parts used,method of preparation medicinal \& nutraceutical importance of LADs.

\begin{tabular}{|c|c|c|c|c|c|c|c|c|c|}
\hline \multirow[b]{2}{*}{$\begin{array}{l}\text { S. } \\
\text { No. }\end{array}$} & \multirow[b]{2}{*}{$\begin{array}{l}\text { Family } \\
\text { Botanical } \\
\text { name/ } \\
\text { Common } \\
\text { name/Loca } \\
\text { I name. }\end{array}$} & \multirow[b]{2}{*}{$\begin{array}{c}\text { Alt } \\
\text { itu } \\
\text { de } \\
\text { in } \\
\text { (m } \\
\text { ) } \\
\& \\
\text { Lif } \\
\text { e } \\
\text { Fo } \\
\text { rm }\end{array}$} & \multirow[b]{2}{*}{$\begin{array}{c}\text { Name } \\
\text { of } \\
\text { Alcohol }\end{array}$} & \multicolumn{2}{|c|}{ Status } & \multirow[b]{2}{*}{$\begin{array}{c}\text { Parts } \\
\text { used }\end{array}$} & \multirow[b]{2}{*}{$\begin{array}{c}\text { Ingredi } \\
\text { ents }\end{array}$} & \multirow[b]{2}{*}{ Process } & \multirow[b]{2}{*}{$\begin{array}{l}\text { Medicinal\& } \\
\text { nutraceutical } \\
\text { importance }\end{array}$} \\
\hline & & & & $\begin{array}{c}\text { Abun } \\
\text { dant } \\
(\text { Ab)/ } \\
\text { Rare } \\
(\mathbf{R})\end{array}$ & $\begin{array}{l}\text { Wild } \\
\text { (W)/ } \\
\text { Cultivat } \\
\text { ed } \\
(\mathbf{C}) / \text { Bot } \\
\text { h }(\mathbf{B})\end{array}$ & & & & \\
\hline & \multicolumn{9}{|c|}{ Amranthaceae } \\
\hline 1. & $\begin{array}{l}\text { Celosia } \\
\text { argentea/Bi } \\
\text { thu }\end{array}$ & $\begin{array}{l}16 \\
00 \\
\mathrm{~m}( \\
\mathrm{H})\end{array}$ & $\begin{array}{l}\text { Bithu ki } \\
\text { Shraab/ } \\
\text { Darru }\end{array}$ & (R) & (B) & $\mathrm{Sd}$ & $\begin{array}{l}\mathrm{Jg}, \mathrm{Sd} \text { of } \\
\text { plant }\end{array}$ & $\begin{array}{l}\text { Step1. Wash } 1 \mathrm{~kg} \text { Celosia sd } \\
\text { properly \& keep it in water for } \\
7-8 \mathrm{~h} \text {. } \\
\text { Step2. Now drain the water } \\
\text { and dry the Celosia sd for } \\
\text { another } 7-8 \mathrm{~h} \text {. }\end{array}$ & $\begin{array}{l}\text { Md: Sd are useful in the } \\
\text { treatment of bloody stool, } \\
\text { haemorrhoid \& uterine } \\
\text { bleeding, leucorrhoea, } \\
\text { dysentery, diarrhoea, } \\
\text { bloodshot eyes, blurring } \\
\text { of vision, cataracts and } \\
\text { hypertension. These are }\end{array}$ \\
\hline
\end{tabular}

Copyright (c) 2020-2021, Journal of Scientific Research in Medical and Biological Sciences (JSRMBS), 


\begin{tabular}{|c|c|c|c|c|c|c|c|c|c|}
\hline & & & & & & & & $\begin{array}{l}\text { Step } 3 \text { Repeat the process to } \\
\text { convert starch into sugar, make } \\
\text { sure that Sd are sprouted. } \\
\text { Step } 4 \text {. keep sd in paper bags } \\
\text { for } 1-2 \text { weeks. } \\
\text { Step } 5 \text { Now we can use them to } \\
\text { make alcohol through } \\
\text { distillation process. }\end{array}$ & $\begin{array}{l}\text { widely used by locals for } \\
\text { the treatment of diabetes } \\
\text { mellitus. }\end{array}$ \\
\hline & \multicolumn{9}{|c|}{ Anacardaceae } \\
\hline \multirow[t]{2}{*}{2.} & $\begin{array}{l}\text { Mangifera } \\
\text { indica } \\
\text { /mango/Aa } \\
m\end{array}$ & $\begin{array}{l}12 \\
00 \\
\mathrm{~m} \\
(\mathrm{~T})\end{array}$ & $\begin{array}{l}\text { Aam ki } \\
\text { desi } \\
\text { Daaru/w } \\
\text { ine }\end{array}$ & $(\mathrm{Ab})$ & (B) & Fr & $\begin{array}{l}\text { Fr, } \\
\text { Jg/sugar } \\
\text { Yeast }\end{array}$ & $\begin{array}{l}\text { Step1 Take } 1 \mathrm{~kg} \text { Mangifera, } \\
\text { Cut into small piece \& add } \\
\text { jaggery } \\
\text { Step } 2 \text {. Mesh pieces manually } \\
\text { and add about } 750 \mathrm{ml} \text { water to } \\
\text { it. Mix well till the jaggery get } \\
\text { dissolved. } \\
\text { Step } 3 \text {. Add } 1-2 \text { spoon of yeast } \\
\text { and mix well .and pour into } \\
\text { bottle. } \\
\text { Step } 4 \text {. leave it for } 15 \text { days and } \\
\text { after that open the cap and } \\
\text { drain into another container. } \\
\text { Step } 5 \text { Add more Jg/sugar } \\
\text { make sure it is dissolved well. } \\
\text { Step } 6 \text { Again store it in bottle } \\
\text { and leave it for } 15 \text { days. } \\
\text { After that it is ready to serve. }\end{array}$ & $\begin{array}{l}\text { Md: The Rt and Br are } \\
\text { usdful in vitiated } \\
\text { conditions of pitta, } \\
\text { metrorrhagia, } \\
\text { colonorrhagia, } \\
\text { pneumorrhagia, } \\
\text { lucorrhoea, syphilis, } \\
\text { wounds, ulcers, vomiting, } \\
\text { uteritis, diarrhoea, } \\
\text { dysentery, diphtheria and } \\
\text { rheumatism. } \\
\text { Nc: Water-78.9-82.8g; } \\
\text { Ashes-0.34-0.52G; Total } \\
\text { lipid-0.30-0.53g; Total } \\
\text { protein-0.36-0.40g; Total } \\
\text { carbohydrate-16.20- } \\
\text { 17.18g; Total dietary } \\
\text { fibres-0.85-1.06;Energy } \\
\text { (kcal)- (Tharanathan } \text { et } \\
\text { al., 2006). }\end{array}$ \\
\hline & \multicolumn{9}{|l|}{ Apiaceae } \\
\hline 3. & $\begin{array}{l}\text { Angelica } \\
\text { glaucal } \\
\text { Smooth } \\
\text { Angelica/ } \\
\text { Chora }\end{array}$ & $\begin{array}{l}25 \\
00- \\
30 \\
00 \\
\mathrm{~m}( \\
\mathrm{H})\end{array}$ & $\begin{array}{l}\text { Chora } \\
\text { Ki } \\
\text { shraab/ } \\
\text { Darru }\end{array}$ & (R) & $(\mathrm{W})$ & $\mathrm{Rt}$ & $\begin{array}{l}\mathrm{Jg}, \mathrm{Rt} \text { of } \\
\text { plant }\end{array}$ & $\begin{array}{l}\text { Step1. Take } 2 \mathrm{~kg} \text { thoroughly } \\
\text { washed Rt of Angelical } \\
\text { Step2. Soak Rt \& jaggery for } \\
\text { 1-2 weeks.in air tight } \\
\text { container. } \\
\text { Step3. Strain the liquid into } \\
\text { boiling pot and turn on the } \\
\text { stove. } \\
\text { Step } 4 \text { Through fermentation } \\
\text { /distillation process we can } \\
\text { pour drink }\end{array}$ & $\begin{array}{l}\text { Md:The Rt is considered } \\
\text { pungent, aromatic, } \\
\text { stomachic, tonic, } \\
\text { stimulant, carminative, } \\
\text { diphoretic, and diuretic. } \\
\text { These are helpful to } \\
\text { increases appetite and } \\
\text { combined with tonic are } \\
\text { given in typhoid } \\
\text { conditions, bronchitis, } \\
\text { flatulence, colic and in } \\
\text { stomach pain. }\end{array}$ \\
\hline & \multicolumn{9}{|l|}{ Araceae } \\
\hline 4. & $\begin{array}{l}\text { Arisaema } \\
\text { jacquemont } \\
\text { ii Cobra } \\
\text { lily/ } \\
\text { Chichyda }\end{array}$ & $\begin{array}{l}20 \\
00- \\
40 \\
00 \\
\mathrm{~m}( \\
\mathrm{H})\end{array}$ & $\begin{array}{l}\text { Chichyd } \\
\text { a ki } \\
\text { shraab/ } \\
\text { Darru }\end{array}$ & (R) & $(\mathrm{W})$ & $\mathrm{Rt}$ & $\begin{array}{l}\text { Jg, Rt of } \\
\text { plant }\end{array}$ & Same as Angelica glauca & $\begin{array}{l}\text { Md: Tu are considered } \\
\text { anti-insect, antimalarial } \\
\text { and anti-cancer. Rt are } \\
\text { antioxidant, antifungal } \\
\text { and antibacterial. } \\
\text { Underground parts are } \\
\text { ground with edible oil to } \\
\text { form a paste, which is } \\
\text { used for massage } \\
\text { purposes in order to } \\
\text { regain the muscular } \\
\text { strength and in skin } \\
\text { problems. } \\
\text { Nc:Rt (chloroform) } \\
\text { contain phenol, flavonoid, } \\
\text { Terpenes, saponins and } \\
\text { glycoside (Tanveer et al.( } \\
\text { 2014) }\end{array}$ \\
\hline 5. & $\begin{array}{l}\text { Arisaema } \\
\text { tortuosum/ }\end{array}$ & $\begin{array}{l}80 \\
0-\end{array}$ & $\begin{array}{l}\text { Cobra } \\
\text { chali ki }\end{array}$ & (R) & $(\mathrm{W})$ & $\mathrm{Rt}$ & $\begin{array}{l}\text { Jg, Rt of } \\
\text { plant }\end{array}$ & Same as Angelica glauca & $\begin{array}{l}\text { Md:Tu are useful to cure } \\
\text { various ailments related to }\end{array}$ \\
\hline
\end{tabular}




\begin{tabular}{|c|c|c|c|c|c|c|c|c|c|}
\hline & $\begin{array}{l}\text { Cobra Fl/ } \\
\text { Kadae ki } \\
\text { chhali }\end{array}$ & $\begin{array}{l}30 \\
00 \\
\mathrm{~m}( \\
\mathrm{H})\end{array}$ & $\begin{array}{l}\text { shraab } \\
\text { /Darru }\end{array}$ & & & & & & $\begin{array}{l}\text { digestive tract like } \\
\text { constipation, indigestion, } \\
\text { abdominal pain, } \\
\text { dysentery, bone fracture . } \\
\text { Corm boiled with } \\
\text { tamarind taken internally } \\
\text { to cure piles. }\end{array}$ \\
\hline & \multicolumn{9}{|l|}{ Arecaceae } \\
\hline 6. & \multicolumn{9}{|l|}{ Asteraceae } \\
\hline 7. & $\begin{array}{l}\text { Arctium } \\
\text { lappa/Grea } \\
\text { t Burdock/ } \\
\text { Jangali } \\
\text { kuth }\end{array}$ & $\begin{array}{l}70 \\
0- \\
35 \\
00 \\
\mathrm{~m}( \\
\mathrm{Sh})\end{array}$ & $\begin{array}{l}\text { Jangali } \\
\text { kuth } k i \\
\text { shraab }\end{array}$ & (R) & $(\mathrm{W})$ & $\mathrm{Rt}$ & $\begin{array}{l}\text { Rt, Jg, } \\
\text { Cinnam } \\
\text { on, }\end{array}$ & $\begin{array}{l}\text { Step1 Cut Arctium Rt in small } \\
\text { pieces and boil. } \\
\text { Step2 Put it in a big jar, Add } \\
\text { some herbs of your choice like } \\
\text { cannabis, Centella, cloves } \\
\text { black pepper \& yeast in it. } \\
\text { Step3 Add water and mix well. } \\
\text { Step4 Keep it in rest for } 3 \\
\text { weeks. Fermentation process } \\
\text { takes place. } \\
\text { Step } 5 \text { After } 3 \text { weeks drain out } \\
\text { water in another boiling pot. } \\
\text { Step6 Put container on stove } \\
\text { and through distillation process } \\
\text { we can collect fresh apple } \\
\text { daaru. (Throw away first } 10 \\
\text { ml) }\end{array}$ & $\begin{array}{l}\text { Md:Rt are effective in } \\
\text { curing infection, diseases, } \\
\text { burns, bruises, boils etc } \\
\text { The dried Rt of one year } \\
\text { old plants is the official } \\
\text { herb, but the leaves and } \\
\text { Frs can also be used. }\end{array}$ \\
\hline & \multicolumn{9}{|c|}{ Berberidaceae } \\
\hline 8. & $\begin{array}{l}\text { Berberis } \\
\text { lycium/ } \\
\text { Indian } \\
\text { Lycium/ } \\
\text { kasmalae }\end{array}$ & $\begin{array}{l}\text { Up } \\
\text { to } \\
25 \\
00 \\
\mathrm{~m}( \\
\mathrm{Sh})\end{array}$ & $\begin{array}{l}\text { Kasmale } \\
\text { ki } \\
\text { shraab/ } \\
\text { Darru }\end{array}$ & $(\mathrm{Ab})$ & $(\mathrm{W})$ & $\mathrm{Rt}$ & $\begin{array}{l}\mathrm{Jg}, \mathrm{Rt} \text { of } \\
\text { plant }\end{array}$ & $\begin{array}{l}\text { Step. } 1 \text { Take a big container } \\
\text { and keep the jaggery and } \\
\text {. Berberis Rt in water for } 1 \\
\text { week. If we are taking } 2 \mathrm{~kg} \text { of } \\
\text { jaggery and Rt then put it in } 10 \\
\text { lit. of water. } \\
\text { Step. } 2 \text { After a week drain } \\
\text { water into boiling pot and let } \\
\text { the Rt remain in it.( Spices \& } \\
\text { herbs like cloves, cardamom, } \\
\text { cannabis, Centella can be } \\
\text { added) } \\
\text { Step. } 3 \text {. Put these containers } \\
\text { on stove and through }\end{array}$ & $\begin{array}{l}\text { Md: Rt decoction } \\
\text { "Rasaunt" is used to cure } \\
\text { eye infections. These are } \\
\text { also used in splenic } \\
\text { troubles; tonic, febrifuge; } \\
\text { intestinal astringent; good } \\
\text { for cough, chest and } \\
\text { throat troubles, eye sores } \\
\text { and itching of the eyes; } \\
\text { piles, menorrhagia; useful } \\
\text { in chronic diarrhea. } \\
\text { Nc: Rt. possess dry } \\
\text { matter, } 61.2 \% \text {; moisture, } \\
20.5 \% \text {; protein, } 4.5 \% ; \text { fat, }\end{array}$ \\
\hline
\end{tabular}




\begin{tabular}{|c|c|c|c|c|c|c|c|c|c|}
\hline & & & & & & & & $\begin{array}{l}\text { distillation process you will } \\
\text { get fresh desi darru }\end{array}$ & $\begin{array}{l}2.6 \% ; \text { sugar, } 3.5 \% ; \\
\text { fibre, } 2.5 \% \text {; and Vit, C, } \\
0.3 \% \text {. Berberine and } \\
\text { palmitine are found in Rt } \\
\text { in concentration of } 4.5 \\
\text { and } 3.1 \% \text {, respectively. } \\
\text { (Gulfraz et al., 2004) }\end{array}$ \\
\hline & \multicolumn{9}{|c|}{ Combertaceae } \\
\hline 9. & $\begin{array}{l}\text { Terminalia } \\
\text { chebula } \\
\text { /Black } \\
\text { Myrobalan/ } \\
\text { Harad }\end{array}$ & $\begin{array}{l}15 \\
00 \\
\mathrm{~m} \\
(\mathrm{~T})\end{array}$ & $\begin{array}{l}\text { Harad } \\
\text { ki } \\
\text { shraab/ } \\
\text { Thardal } \\
\text { Darru }\end{array}$ & (Ab) & (B) & $\mathrm{fr}$ & $\begin{array}{l}\mathrm{Jg}, \mathrm{Fr} \text { of } \\
\text { plant }\end{array}$ & $\begin{array}{l}\text { Step 1. Terminalia Fr are } \\
\text { crushed in pestle and mortar, } \\
\text { mixed with jaggery and yeast } \\
\text { and placed inside a airtight } \\
\text { container for fermentation. } \\
\text { Step 2. After 7-15 days this } \\
\text { fermented product is ready for } \\
\text { distillation. (Distillation is } \\
\text { usually performed in a special } \\
\text { setup made up of two container } \\
\text { or vessel plus outlet for } \\
\text { distillate collection. Bottom } \\
\text { vessel is meant for fermented } \\
\text { mixture and top vessel or } \\
\text { covering for vapours } \\
\text { collection. ) } \\
\text { Step3- Covering vessel is } \\
\text { usually sealed with dough to } \\
\text { avoid any leakage. } \\
\text { Step4- Distillate(alcoholic } \\
\text { drink) is collected in glass } \\
\text { bottles through outlet. }\end{array}$ & $\begin{array}{l}\text { Md:Fr are laxative and } \\
\text { digestive, and constitute } \\
\text { one of the 'Triphala' of } \\
\text { Ayurveda. Fr are } \\
\text { astringent, sweet, acrid, } \\
\text { bitter, sour, thermogenic, } \\
\text { anodyne, anti- } \\
\text { inflammatory, vulnerary, } \\
\text { alterant, stomachic, and } \\
\text { laxative, purgative, } \\
\text { carminative, digestive, } \\
\text { anthelmintic, cardiotonic, } \\
\text { aphrodisiac, antiseptic, } \\
\text { febrifuge, depurative and } \\
\text { tonic. Thus helpful to cure } \\
\text { a wide range of diseases. } \\
\text { Nc: Chebulin from } \\
\text { flowers. Palmitic, oleic, } \\
\text { linoleic, arachidic stearic } \\
\text { and behenic acids from } \\
\text { fruit kernels }{ }^{1} \text {. Fruits } \\
\text { contain about } 30 \% \text { of an } \\
\text { astringent substance; } \\
\text { astringency is due to the } \\
\text { characteristic principle } \\
\text { chebulic acid. Also } \\
\text { contain tannic acid } 20- \\
40 \%, \text { Gallic acid, resin } \\
\text { etc. and a purgative } \\
\text { glycoside of } \\
\text { anthraquinone derivative. } \\
\text { Chebulin exhibited } \\
\text { antispasmodic action on } \\
\text { smooth muscle similar to } \\
\text { papaverine }{ }^{1} \text {.( Purohit et } \\
\text { al. (2009) }\end{array}$ \\
\hline
\end{tabular}

Abbreviations used: $H=$ Herb; $T=$ Tree; Sh=Shrub; St=Stem; Fr=Fruit;Bk=Bark; Rh=Rhizome; Sd=Seed; $T s=$ Tender shoot; Bd=Buds Tu= tuber; Ap=Aerial Part; Lf=Leaf; Fl= Flower; Ab=Abundant; R= Rare; $W=$ Wild $; C=$ Cultivated $B=$ Both;Jg=Jeggery:Md-Medicinal: $:$ c =Nutraceutical, 


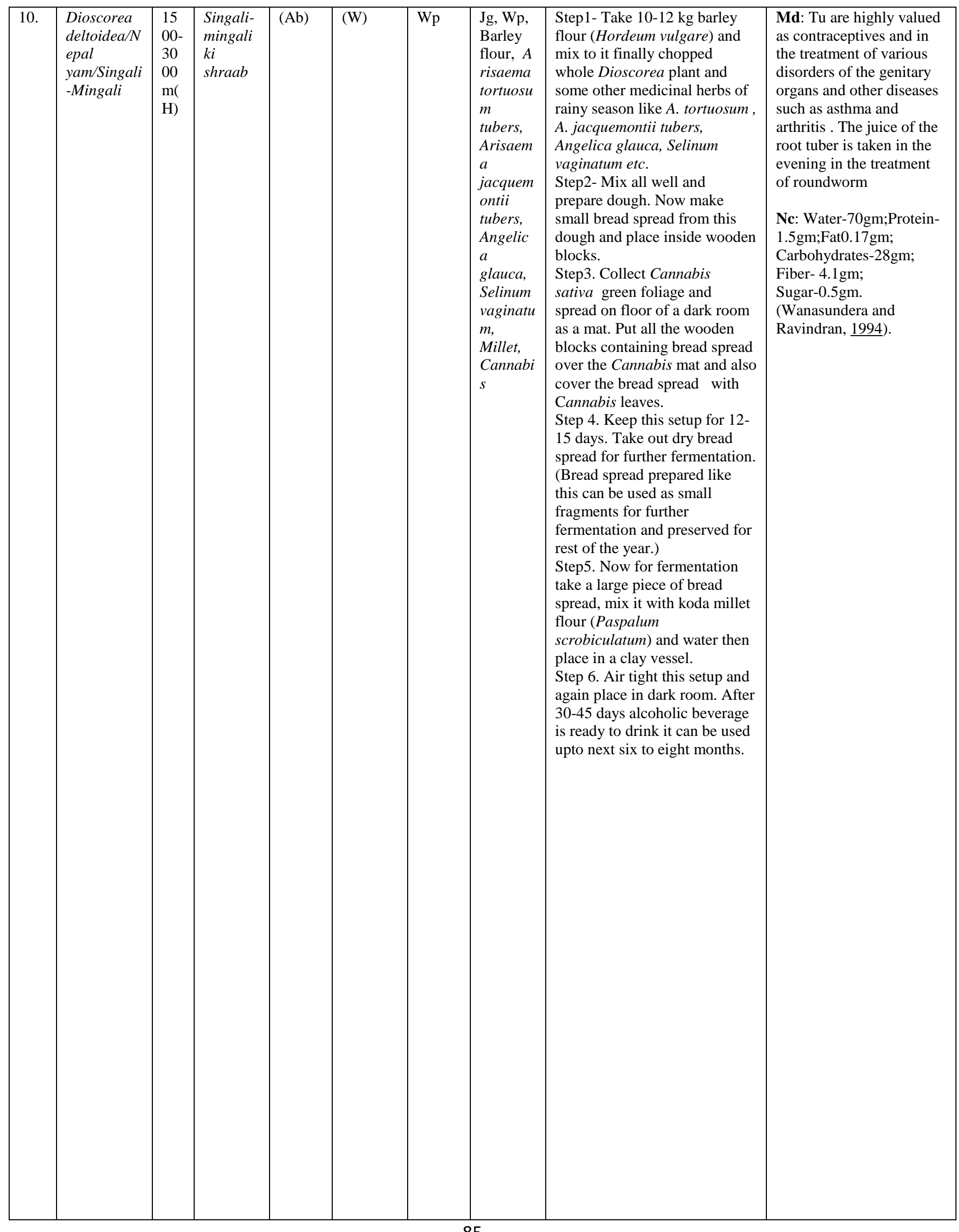




\begin{tabular}{|c|c|c|c|c|c|c|c|c|c|}
\hline & \multicolumn{9}{|l|}{ Fabaceae } \\
\hline 11. & $\begin{array}{l}\text { Acacia } \\
\text { nilotica/Gu } \\
\text { m Arabic } \\
\text { tree/Babool }\end{array}$ & $\begin{array}{l}\text { Up } \\
\text { to } \\
60 \\
0 \\
\text { m( } \\
\text { T) }\end{array}$ & $\begin{array}{l}\text { Kikkare } \\
\text { ki Desi } \\
\text { shraab } \\
\text { /Tharda } \\
\text { /Darru }\end{array}$ & $(\mathrm{Ab})$ & $(\mathrm{W})$ & $\mathrm{Br}$ & $\begin{array}{l}\mathrm{Jg}, \mathrm{Br} \text { of } \\
\text { plant, }\end{array}$ & Same as Berberis lycium. & $\begin{array}{l}\text { Md: The Rt are used } \\
\text { against cancers } \\
\text { tuberculosis and } \\
\text { indurations of liver and } \\
\text { spleen. Plant is } \\
\text { antimutagenic, anti } \\
\text { bacterial, anticancer, } \\
\text { astringent \& shows anti- } \\
\text { microbial activity } \\
\text { Nc: Astringent, emollient, } \\
\text { liver tonic, antipyretic and } \\
\text { antiasthmatic.(Kalaivani } \\
\text { and Mathew, 2010 } \\
\text { Kalaivani and Mathew, } \\
\text { 2010; Shittu, } \\
\text { 2010;(Baravkar et al., 2008) }\end{array}$ \\
\hline 12. & $\begin{array}{l}\text { Bauhinia } \\
\text { variegatel } \\
\text { Orchid } \\
\text { tree/Kachn } \\
\text { ar }\end{array}$ & $\begin{array}{l}\mathrm{Up} \\
\text { to } \\
17 \\
00 \\
\mathrm{~m} \\
(\mathrm{~T})\end{array}$ & $\begin{array}{l}\text { Karyale } \\
\text { ki } \\
\text { shraab/ } \\
\text { Thardal } \\
\text { Darru }\end{array}$ & $(\mathrm{Ab})$ & $(\mathrm{W})$ & $\mathrm{Br}, \mathrm{Fl}$ & $\begin{array}{l}\mathrm{Jg}, \mathrm{Br} \\
\text { and Fl } \\
\text { of plant, } \\
\text { cinnamo } \\
\mathrm{n}, \\
\text { cardamo } \\
\text { m, } \\
\text { cloves }\end{array}$ & $\begin{array}{l}\text { Step1 Take out } 1 \mathrm{~kg} \text { of } \\
\text { Bauhinia } \mathrm{Fl} \text { and } \mathrm{Br} \& \text { wash } \\
\text { properly. } \\
\text { Step2 Dip them into water \& } \\
\text { add Cinnamon sticks, cloves, } \\
\text { cardamom and leave it for 10- } \\
12 \mathrm{~h} \text {. } \\
\text { Step3 Boil this with } 1 \mathrm{~kg} \\
\text { jaggery in } 1 \text { lit water. Wait till } \\
\text { it cools down. } \\
\text { Step } 4 \text {. Take a bowl, add some } \\
\text { sugar solution \& } 2 \text { spoon of } \\
\text { yeast. Leave it for } 30 \text { min } \\
\text { Step 5. Add sugar solution \& } \\
\text { yeast in the jar. } \\
\text { Step6 After few hours } \\
\text { fermenting will starts and } \\
\text { alcohol is ready to collect. }\end{array}$ & $\begin{array}{l}\text { Md:The Br of this plant is } \\
\text { considered acrid, sweet, } \\
\text { appetising, cooling, } \\
\text { astringent to the bowels. It } \\
\text { is helpful to cures } \\
\text { biliousness, "Kapha", } \\
\text { leukoderma, anal troubles, } \\
\text { tuberculous glands, } \\
\text { cough, asthma, diseases of } \\
\text { the blood, ulcers, vaginal } \\
\text { discharges; anthelmintic; } \\
\text { used in strangury, thirst, } \\
\text { burning sensation. } \\
\text { decoction of Rt is given } \\
\text { in dyspepsia and } \\
\text { flatulency. The dried buds } \\
\text { are used in piles and } \\
\text { dysentery. They are } \\
\text { considered cool and } \\
\text { astringent, and are useful } \\
\text { in diarrhoea and worms. } \\
\text { Nc:100 g Fl buds contains } \\
\text {; moisture, } 78.9 \text { g; protein, } \\
1.8 \text { g; fat, } 0.2 \mathrm{~g} \text {; fibre, } 1.3 \text {; } \\
\text { carbohydrates, } 17.8 \text {; total } \\
\text { minerals ash, } 1.3 \mathrm{~g} \text {; Ca, } \\
70.1 \text { mg; P, } 74.2 \text { mg \& Fe, } \\
6.1 \text { mg. (Anonymous, } \\
1988 \text { ) }\end{array}$ \\
\hline & \multicolumn{9}{|c|}{ Hypericaceae } \\
\hline 13. & $\begin{array}{l}\text { Hypericum } \\
\text { perforatum/ } \\
\text { St. John's } \\
\text { Wort/Basa } \\
\text { nti }\end{array}$ & $\begin{array}{l}20 \\
00- \\
32 \\
00 \\
\mathrm{~m}( \\
\mathrm{Sh})\end{array}$ & $\begin{array}{l}\text { Basanti } \\
\text { ki } \\
\text { shraab/ } \\
\text { Darru }\end{array}$ & $(\mathrm{Ab})$ & $(\mathrm{W})$ & $\mathrm{Fl}$ & $\begin{array}{l}\mathrm{Jg}, \mathrm{Fl} \text { of } \\
\text { plant }\end{array}$ & Same as Berberis lycium. & $\begin{array}{l}\text { Md: Fl are considered } \\
\text { antidepressant, } \\
\text { antispasmodic, stimulates } \\
\text { bile flow, astringent, } \\
\text { sedative \& antiviral. An } \\
\text { infusion of the Fl in olive } \\
\text { oil is applied externally to } \\
\text { wounds, sores, ulcers, } \\
\text { swellings, rheumatism etc. } \\
\text { This plant is helpful in } \\
\text { treating a wide range of } \\
\text { disorders, including } \\
\text { pulmonary complaints, }\end{array}$ \\
\hline
\end{tabular}




\begin{tabular}{|c|c|c|c|c|c|c|c|c|c|}
\hline & & & & & & & & & $\begin{array}{l}\text { bladder problems, } \\
\text { diarrhoea and nervous } \\
\text { depression. }\end{array}$ \\
\hline & \multicolumn{9}{|l|}{ Lythraceae } \\
\hline \multirow[t]{2}{*}{14.} & $\begin{array}{l}\text { Woodfordia } \\
\text { fruticosal } \\
\text { Fire-Flame } \\
\text { bush/ } \\
\text { Dhave }\end{array}$ & $\begin{array}{l}\text { Up } \\
\text { to } \\
18 \\
00 \\
\text { m( } \\
\text { T) }\end{array}$ & $\begin{array}{l}\text { Dhave } \\
\text { ki Desi } \\
\text { Shraab/ } \\
\text { Darru }\end{array}$ & $(\mathrm{Ab})$ & $(\mathrm{W})$ & $\mathrm{Br}$ & $\begin{array}{l}\mathrm{Jg}, \mathrm{Br} \text { of } \\
\text { plant }\end{array}$ & $\begin{array}{l}\text { Step1, Cut Woodfordia Br in } \\
\text { small pieces. } \\
\text { Step2. Put it in a big jar \& add } \\
\text { some, cloves black pepper, } \\
\text { yeast in it. } \\
\text { Step 3. Add some water and } \\
\text { mix it well. } \\
\text { Step } 4 \text {. Keep it in rest for about } \\
3 \text { weeks for fermentation. } \\
\text { Step 5. After } 3 \text { weeks drain out } \\
\text { water in another container. } \\
\text { Step 6. Put container on stove } \\
\text { and through distillation process } \\
\text { we can collect fresh } \\
\text { daaru.(throw away first } 10 \mathrm{ml} \\
\text { ) }\end{array}$ & $\begin{array}{l}\text { Md: Almost all the parts } \\
\text { of dhave plant viz Fls, } \\
\text { Frs, Rt, leaves \& buds } \\
\text { mixed with pedicels \& } \\
\text { thinner twigs possess } \\
\text { valuable medicinal } \\
\text { properties like regulate } \\
\text { liver disorder, manage } \\
\text { gynological disorder \& } \\
\text { poultice applied on } \\
\text { forehead relieves } \\
\text { headache }\end{array}$ \\
\hline & \multicolumn{9}{|l|}{ Myricaceae } \\
\hline \multirow[t]{2}{*}{15.} & $\begin{array}{l}\text { Myrica } \\
\text { esculenta/K } \\
\text { afal }\end{array}$ & $\begin{array}{l}90 \\
0- \\
18 \\
00 \\
\mathrm{~m}( \\
\mathrm{T})\end{array}$ & $\begin{array}{l}\text { Kafal ki } \\
\text { shraab/ } \\
\text { Darru }\end{array}$ & $(\mathrm{Ab})$ & $(\mathrm{W})$ & $\mathrm{Br}$ & $\begin{array}{l}\mathrm{Jg}, \mathrm{Br} \text { of } \\
\text { plant }\end{array}$ & Same as Arisaema tortuosum. & $\begin{array}{l}\text { Md: Powered Sd \& Fr are } \\
\text { useful against stomach } \\
\text { disorders. Br is } \\
\text { astringent, stimulant, } \\
\text { carminative, tonic and } \\
\text { resolvent, used in fever, } \\
\text { asthma, diarrhea, chronic } \\
\text { bronchitis, dysentery and } \\
\text { diuresis. } \\
\text { Nc: Fr contains crude } \\
\text { proteins - } 1.3 \% \text {, } \\
\text { Carbohydrates-16.13\%, } \\
\text { Crude fibres-3.4\% and ash } \\
\text { content-1.25\% . Minerals } \\
\text { as Ca, Mg, K, and P } 1.0, \\
8.4,1.98 \text { and } 0.24 \mathrm{mg} / \mathrm{gm} \\
\text { respectively }\end{array}$ \\
\hline & \multicolumn{9}{|l|}{ Poaceae } \\
\hline 16. & $\begin{array}{l}\text { Hordeum } \\
\text { vulgare/Ba } \\
\text { rley/jau }\end{array}$ & $\begin{array}{l}45 \\
00 \\
\mathrm{~m}( \\
\mathrm{T})\end{array}$ & $\begin{array}{l}\text { Jau ki } \\
\text { shraab } \\
\text { /Desi } \\
\text { Daaru }\end{array}$ & $(\mathrm{Ab})$ & (C) & $\mathrm{Sd}$ & $\begin{array}{l}\text { Jau Sd, } \\
\text { Jg }\end{array}$ & $\begin{array}{l}\text { Step1 Firstly wash } 1 \mathrm{~kg} \text { barley } \\
\text { properly and after that keep it } \\
\text { in water for } 8 \mathrm{hrs} \text {. } \\
\text { Step2 Now drain the water and } \\
\text { dry the barley sds for } 8 \mathrm{hrs} \text {. } \\
\text { Step3 Again do in the same } \\
\text { way soak in water and dry the } \\
\text { sds properly. (In this process } \\
\text { starch converted into sugar) } \\
\text { make sure they are sprouted. } \\
\text { Step-4 keep sd in paper bags } \\
\text { for } 1-2 \text { weeks. } \\
\text { Step-5 Now we can use them } \\
\text { to make alcohol through } \\
\text { fermentation process. }\end{array}$ & $\begin{array}{l}\text { Md: Sd are digestive, } \\
\text { emollient, nutritive, } \\
\text { febrifuge and stomachic. } \\
\text { The germinating Sd has a } \\
\text { hypoglycaemic, anti- } \\
\text { tumorous \& helpful in } \\
\text { weight management. } \\
\text { Nc: Whole grain consists } \\
\text { of about } 65-68 \% \text { starch, } \\
10-17 \% \text { protein, } 2 \text { - } 3 \% \\
\text { free lipids, } 4-9 \% \beta- \\
\text { glucans and } 1.5-2.5 \% \\
\text { minerals Madhusweta et } \\
\text { al. (2016) }\end{array}$ \\
\hline 17. & $\begin{array}{l}\text { Oryza } \\
\text { satival } \\
\text { rice/chawal }\end{array}$ & $\begin{array}{l}25 \\
00 \\
\mathrm{~m} \\
(\mathrm{H})\end{array}$ & $\begin{array}{l}\text { Chawal } \\
\text { ki } \\
\text { shraab/ } \\
\text { Lugadi }\end{array}$ & $(\mathrm{Ab})$ & (C) & $\mathrm{Sd}$ & $\begin{array}{l}\text { Phaaph ( } \\
\text { mixture } \\
\text { of } \\
\text { barley }\end{array}$ & $\begin{array}{l}\text { Step1 cook } 5 \text { cups of rice, add } \\
25 \text { gms of Phaaph or yeast and } \\
\text { mix it well. } \\
\text { Step2 Transfer mixed rice to a } \\
\text { air tight container. }\end{array}$ & $\begin{array}{l}\text { Md: Sd are nutritive, } \\
\text { soothing, tonic \& } \\
\text { diuretic, helpful to } \\
\text { reduces lactation, urinary } \\
\text { dysfunction \& improves }\end{array}$ \\
\hline
\end{tabular}




\begin{tabular}{|c|c|c|c|c|c|c|c|c|c|}
\hline & & & & & & & $\begin{array}{l}\text { and rice) } \\
\text {,yeast }\end{array}$ & $\begin{array}{l}\text { Step3 Store in a closed place } \\
\text { for } 6 \text { days. } \\
\text { Step4 Now add } 2 \text { lit. of water } \\
\text { again mix it properly. } \\
\text { Step } 5 \text { Store it for } 2 \text { days in a } \\
\text { closed place. } \\
\text { Step6 Strain rice water and } \\
\text { ready too use. }\end{array}$ & $\begin{array}{l}\text { digestion and controls } \\
\text { sweating. } \\
\text { Nc: The amount fat, } \\
\text { soluble vitamins A and } \\
D \text { in rice is } \\
\text { negligible. But vitamin E } \\
\text { content of whole rice is } \\
\text { considerable Husked rice } \\
\text { has a high content of } \\
\text { vitamin B, at least } \\
1 / 10 \text { thof dried feast. The } \\
\text { riboflavin content is low } \\
\text { and vitamin C is absent. } \\
\text { (Sotelo et. al 1990) }\end{array}$ \\
\hline 18. & $\begin{array}{l}\text { Paspalum } \\
\text { scorbiculat } \\
\text { um/ Kodra }\end{array}$ & $\begin{array}{l}\text { Up } \\
\text { to } \\
13 \\
00 \\
\mathrm{~m}( \\
\mathrm{H})\end{array}$ & $\begin{array}{l}\text { Kodre ki } \\
\text { Desi } \\
\text { shraab/ } \\
\text { Thardal } \\
\text { Darru }\end{array}$ & (R) & (C) & $\mathrm{Sd}$ & $\begin{array}{l}\mathrm{Jg}, \mathrm{Sd} \\
\text { of plant }\end{array}$ & Same as Hordeum vulgare. & $\begin{array}{l}\text { Md: Sd are considered } \\
\text { Anti-cholesterol and anti- } \\
\text { hypertension. These are } \\
\text { helpful in weight, } \\
\text { cholesterol \& sugar } \\
\text { management. } \\
\text { Nc: } 100 \mathrm{~g} \text { grain contains } \\
\text { carbohydrate, moisture, } \\
\text { fiber, protein and fat is } \\
64.3 \mathrm{~g}, 11.2 \%, 8.3 \mathrm{~g}, 8.1 \mathrm{~g} \\
\text { and } 1.3 \mathrm{~g} \text { respectively, } \\
\text { minerals like } \mathrm{P}, \text { Ca and Fe } \\
\text { is } 16 \mathrm{mg}, 32 \mathrm{mg} \text { and } 0.5 \mathrm{mg} \\
\text { respectively ((Muragod et } \\
\text { al., 2019) }\end{array}$ \\
\hline 19. & $\begin{array}{l}\text { Zea mays } \\
\text { /Maize/chal } \\
\text { i/ Makki }\end{array}$ & $\begin{array}{l}\text { Up } \\
\text { to } \\
30 \\
0 \\
\mathrm{~m}( \\
\mathrm{H})\end{array}$ & $\begin{array}{l}\text { Makki ki } \\
\text { shraab/ } \\
\text { Darru }\end{array}$ & $(\mathrm{Ab})$ & (C) & $\mathrm{Sd}$ & $\begin{array}{l}\mathrm{Jg}, \mathrm{Sd} \\
\text { of the } \\
\text { plant }\end{array}$ & $\begin{array}{l}\text { Step1 Corn is soaked in water } \\
\text { for } 12 \mathrm{hrs} \text {. and then waited till } \\
\text { corn is sprouted and after that } \\
\text { it is dried, grind and boiled in } \\
\text { water. } \\
\text { Step2 Now put grinded paste } \\
\text { in container along with water } \\
\text { add 1lit. water. } \\
\text { Step3 Cook it in moderate } \\
\text { temp and then in high }\left(90-95^{\circ} \mathrm{c}\right) \\
\text { Step4 Now drain water in } \\
\text { another container, and do not } \\
\text { throw remaining corn. } \\
\text { Step5 You can add some more } \\
\text { sugar. } \\
\text { Step6 Fill little amount of corn } \\
\text { in bottle and after that pour } \\
\text { that drained water. And it is } \\
\text { ready to use }\end{array}$ & $\begin{array}{l}\text { Md:Sd are diuretic and a } \\
\text { mild stimulan \& } \\
\text { considered good emollient } \\
\text { poultice for ulcers, } \\
\text { swellings and rheumatic } \\
\text { pains[. Sd are widely } \\
\text { used in the treatment of } \\
\text { cancer, tumours and } \\
\text { warts. } \\
\text { Nc: !00 g grain contains } \\
\text { vitamin C, E, K, } \mathrm{B}_{1}, \mathrm{~B}_{2}, \\
\mathrm{~B}_{3}, \mathrm{~B}_{5}, \mathrm{~B} 6 \text {, folic acid, } \\
\text { selenium, N-p-coumaroyl } \\
\text { tryptamine, and N-ferrulyl } \\
\text { tryptamine. Potassium is a } \\
\text { major nutrient present } \\
\text { (Kumar \& Jhariya, 2013). }\end{array}$ \\
\hline & \multicolumn{9}{|l|}{ Rhamnaceae } \\
\hline 20. & $\begin{array}{l}\text { Ziziphus } \\
\text { mauritianal } \\
\text { Indian } \\
\text { Jujube/ } \\
\text { Baer }\end{array}$ & $\begin{array}{l}18 \\
00 \\
\mathrm{~m}( \\
\mathrm{Sh})\end{array}$ & $\begin{array}{l}\text { Buraadi } \\
\text { /Tharda } \\
\text { / Desi } \\
\text { Darru }\end{array}$ & $(\mathrm{Ab})$ & (W) & $\begin{array}{l}\mathrm{Br}, \mathrm{Rt} \\
\text { and } \mathrm{Fr}\end{array}$ & $\begin{array}{l}\mathrm{Jg}, \mathrm{Br}, \\
\text { Fr and } \\
\text { Rt of the } \\
\text { plant }\end{array}$ & $\begin{array}{l}\text { Step-1 Add Jg and jangli baer, } \\
\text { Rt and Br in water for } 1-2 \\
\text { week. If we are taking } 3 \mathrm{~kg} \text { of } \\
\text { Jg then put it in } 15 \text { lit. of water. } \\
\text { Step-2 After a week drain, that } \\
\text { water into another container } \\
\text { and let the plant extract remain } \\
\text { in it.(other herbs can be added) } \\
\text { Step-3 Put these containers on } \\
\text { stove make sure it is air tighten } \\
\text { and through fermentation }\end{array}$ & $\begin{array}{l}\text { Md: Rt is bitter \& } \\
\text { considered cooling } \\
\text { anodyne \& tonic. These } \\
\text { are useful in vitiated } \\
\text { conditions of pitta, fever, } \\
\text { wounds \& ulcers. The Br } \\
\text { is astringent, constipating, } \\
\text { tonic \& is useful for } \\
\text { dysentery, diarrhea, } \\
\text { gingivitis \& boils. Fr are } \\
\text { sweet, cooling, anodyne } \\
\text { purgative, mucilaginous, }\end{array}$ \\
\hline
\end{tabular}




\begin{tabular}{|c|c|c|c|c|c|c|c|c|c|}
\hline & & & & & & & & $\begin{array}{l}\text { process you will get fresh desi } \\
\text { daru. }\end{array}$ & $\begin{array}{l}\text { pectoral, styptic, } \\
\text { aphrodisiac, invigorating, } \\
\text { depurative, appetizer \& } \\
\text { tonic. } \\
\text { Nc:Moisture-81.6-83.0g; } \\
\text { Protein-0.8g; fat-0.07g; } \\
\text { Fiber-0.60g; } \\
\text { Carbohydrate-17.0g; Tota } \\
\text { sugar-5.4-10.5g; } \\
\text { Reducing sugar1.4-6.2g; } \\
\text { Non-reducing sugar-3.2- } \\
\text { 8.0g; calcium-25.6mg; P- } \\
\text { 26.8mg; iron-0.76-1.8mg; } \\
\text { Carotene-0.021mg; } \\
\text { Thiamine-0.02-0.024mg; } \\
\text { Niacin-0.7- } \\
\text { 0.873mg;Acorbic acid- } \\
\text { 65.8-76.0mg; Fluoride- } \\
\text { 0.1-0.2ppm;pectin-2.2- } \\
\text { 3.4\%.(Morton,1987; } \\
\text { Pareek and } \\
\text { Dhaka,2008;Pareek. et.al } \\
\text {.2009) }\end{array}$ \\
\hline & \multicolumn{9}{|l|}{ Rosaceae } \\
\hline 21. & $\begin{array}{l}\text { Fragaria } \\
\text { vesca } \\
\text { /Jangali } \\
\text { strawberry/ } \\
\text { Bhoomphal }\end{array}$ & $\begin{array}{l}10 \\
00- \\
35 \\
00 \\
\mathrm{~m} \\
(\mathrm{H})\end{array}$ & $\begin{array}{l}\text { Bhoomp } \\
\text { hal ki } \\
\text { shraab }\end{array}$ & $(\mathrm{Ab})$ & (W) & Fr & $\begin{array}{l}\text { Fr, } \\
\text { Cinnam } \\
\text { on, } \\
\text { Mentha, } \\
\text { brahmi }\end{array}$ & Same as Malus domestica & $\begin{array}{l}\text { Md: The Fr are } \\
\text { considered beneficial in } \\
\text { the treatment of liver and } \\
\text { kidney complaints, as wel } \\
\text { as in the treatment of } \\
\text { rheumatism and gout. } \\
\\
\text { Nc: Fr contains water, } 87- \\
88 \text {; sugars } 3.0-4.5 \text {; free } \\
\text { acids, } 1.33-1.65 \text {; and ash } \\
0.6-0.7 \text { per cent }{ }^{2} \text {. (Parmar } \\
\text { et al. }(1982)\end{array}$ \\
\hline 22. & $\begin{array}{l}\text { Malus } \\
\text { domestica/ } \\
\text { Apple/Seb }\end{array}$ & $\begin{array}{l}12 \\
00- \\
35 \\
00 \\
\mathrm{~m}( \\
\mathrm{T})\end{array}$ & $\begin{array}{l}\text { Seb ki } \\
\text { shraab/ } \\
\text { Desi } \\
\text { Daaru }\end{array}$ & $(\mathrm{Ab})$ & (C) & Fr & $\begin{array}{l}\text { Fr, } \\
\text { grapes, } \\
\text { wheat, } \\
\text { cinnamo } \\
\text { n, } \\
\text { cloves, } \\
\text { black } \\
\text { pepper, } \\
\text { yeast }\end{array}$ & $\begin{array}{l}\text { Step1 cut the apple in small } \\
\text { pieces and boil them and grind } \\
\text { it } \\
\text { Step2 Put it in a big jar, you } \\
\text { can also add some grapes } \\
\text {,cinnamon, wheat, cloves black } \\
\text { pepper, yeast in it. } \\
\text { Step3 Add water and mix it } \\
\text { well. } \\
\text { Step4 Keep it in rest for } \\
\text { 3weeks.Fermentation process } \\
\text { takes place. } \\
\text { Step5 After } 3 \text { weeks drain out } \\
\text { water in another container. } \\
\text { Step6 Put container on stove } \\
\text { and through distillation process } \\
\text { we can collect fresh apple } \\
\text { daaru.(throw away first } 10 \mathrm{ml} \\
\text { ) }\end{array}$ & $\begin{array}{l}\text { Md: Fr considered } \\
\text { astringent and laxative. A } \\
\text { ripe raw apple is good for } \\
\text { stomach \& helpful in } \\
\text { digestion. The apple juice } \\
\text { will reduce the acidity of } \\
\text { the stomach, } \\
\text { Nc:100 g Apple contains } \\
\text { multiple vitamins } \\
\text { including vitamin C, E, } \beta \text { - } \\
\text { carotene and essential } \\
\text { mineral elements such as } \\
\mathrm{Ca}, \mathrm{Fe}, \mathrm{K}, \mathrm{Mn}, \mathrm{Zn}, \mathrm{Mg}, \mathrm{Cu} \\
\& \mathrm{~S} \text {. Vitamin } \mathrm{C} \text { is } 5.7 \\
\mathrm{mg} \text {. The mineral content } \\
\text { are } \mathrm{K}(107.25), \mathrm{Ca}(5.80) \text {, } \\
\mathrm{P}(10.87), \mathrm{Mg}(5.07), \mathrm{Na} \\
(0.72), \mathrm{Fe}(0.123), \mathrm{Zn} \\
(0.043), \mathrm{Cu}(0.027) \text {, and } \\
\text { Mn }(0.035) \mathrm{mg} / 100 \mathrm{~g} \text {. } \\
\text { Boyer et al. } 2004 \text {, } \\
\text { Richardson et al. } 2020 \text {, } \\
\text { Nour } \text { et al. } 2010\end{array}$ \\
\hline
\end{tabular}




\begin{tabular}{|c|c|c|c|c|c|c|c|c|c|}
\hline 23. & $\begin{array}{l}\text { Prunus } \\
\text { armeniaca/ } \\
\text { Apricot/Kh } \\
\text { umani } \\
\text { /Khubani }\end{array}$ & $\begin{array}{l}35 \\
00 \\
\mathrm{~m} \\
(\mathrm{~T})\end{array}$ & $\begin{array}{l}\text { Khuman } \\
\text { i ki desi } \\
\text { Daaru }\end{array}$ & $(\mathrm{Ab})$ & (B) & $\mathrm{Fr}$ & $\begin{array}{l}\mathrm{Jg}, \mathrm{fr}, \\
\text { cinnamo } \\
\mathrm{n}, \\
\text { cloves } \\
\text { and } \\
\text { cardamo } \\
\mathrm{m}\end{array}$ & $\begin{array}{l}\text { Step1 Take out } 1 \mathrm{~kg} \text { of apricot } \\
\text { wash them properly. } \\
\text { Step2 Mesh the Frs completely } \\
\text { and then add cinnamon sticks, } \\
\text { cloves, cardamom and leave it } \\
\text { for } 12 \mathrm{hrs} \text {. } \\
\text { Step3 Boil } 1 \mathrm{~kg} \text { sugar in } 1 \text { lit. } \\
\text { water. then wait till it cools } \\
\text { down. } \\
\text { Step4 take a bowl and add } \\
\text { some sugar solution and } 2 \\
\text { spoon of yeast and leave it for } \\
30 \text { min } \\
\text { Step5 Now add sugar solution } \\
\text { as well as yeast that we already } \\
\text { mixed in the first jar. } \\
\text { Step6 Observe few hours later } \\
\text { you can see fermenting/ } \\
\text { distillation starts and we can } \\
\text { collect alcohol. }\end{array}$ & $\begin{array}{l}\text { Md: Fr are nutritious, } \\
\text { cleansing and mildly } \\
\text { laxativ \& are a valuable } \\
\text { addition to the diet } \\
\text { working gently to } \\
\text { improve overall health. } \\
\text { Nc: Carbohydrate } 11-13 \% \\
\text { and provides } 50 \text { kcals of } \\
\text { energy per } 100 \mathrm{~g} \text {. Dietary } \\
\text { fibre1.5-2.4g/100g } \\
\text { The major elements are } \\
\mathrm{K}, \mathrm{P}, \mathrm{Ca}, \mathrm{Mg}, \text { Fe \&Se } \\
\text { while Na, Mn, Zn \& Cu } \\
\text { are also present in small } \\
\text { amounts. vitamins found } \\
\text { in apricot are pro- vitamin } \\
\text { A, } \\
\text { vitamins C, K, E, thiamin } \\
\text { (B1), riboflavin (B2), } \\
\text { niacin (B3), pyridoxine } \\
\text { (B6), folic acid (B9) } \\
\text { and pantothenic acid. } \\
\text { Apricot contains organic } \\
\text { acids i.e. malic acid } \\
\text { (500-900mg/100g) and } \\
\text { citric (30-50mg/100g) } \\
\text { (Leccese } \text { et al. 2007; Ali } \\
\text { et al. 2011; Munzuroglu } \\
\text { et al., 2003; Ali } \text { et } \\
\text { al.,2011; Lichou et al., } \\
\text { 2003; USDA, 2010) } \\
\text { Gurrieri et al. 2001 }\end{array}$ \\
\hline 24. & $\begin{array}{l}\text { Prunus } \\
\text { cerasoides/ } \\
\text { Himalayan } \\
\text { wild } \\
\text { cherry/Bird } \\
\text { cherry/Pajj } \\
\text { a }\end{array}$ & $\begin{array}{l}30 \\
00 \\
\mathrm{~m} \\
(\mathrm{~T})\end{array}$ & $\begin{array}{l}\text { Pajje ki } \\
\text { shraab }\end{array}$ & $(\mathrm{Ab})$ & (B) & $\mathrm{Br}$ & $\begin{array}{l}\text { Br of } \\
\text { plant, } \\
\text { Jg, } \\
\text { cloves, } \\
\text { cardamo } \\
\text { m }\end{array}$ & $\begin{array}{l}\text { Step1 Take a Br of plant and } \\
\text { cut it in small pieces. (Add Jg ) } \\
\text { Step2 Now take a container put } \\
\text { Br pieces and add about } \\
750 \mathrm{ml} \text { water(mix well till the } \\
\text { Jg get dissolved) } \\
\text { Step3 leave it for } 15 \text { days and } \\
\text { after that open the cap and } \\
\text { drain into another container. } \\
\text { Step4 add more Jg/sugar make } \\
\text { sure it is dissolved well. } \\
\text { Step } 5 \text { Again store it in bottle } \\
\text { and leave it for } 15 \text { days. } \\
\text { After that it is ready to serve. }\end{array}$ & $\begin{array}{l}\text { Md:Br is used to check } \\
\text { fever and relieves } \\
\text { stomachache. Heartwood } \\
\text { of this plant is considered } \\
\text { astringent, bitter, acrid } \\
\text { anodyne, refrigerant, } \\
\text { vulnerary, demulcent, } \\
\text { digestive, constipating, } \\
\text { diuretic, emmenagogue, } \\
\text { depurative, antipyretics, } \\
\text { and tonic. It is useful in } \\
\text { vitiated conditions of pitta } \\
\text { and many other diseases. } \\
\text { Nc:Amygdalin, } \\
\text { prunacetin, prunetin, } \\
\text { sakuranetin, puddumetin, } \\
\text { genkwain, padmakastin, } \\
\text { and sauranin have been } \\
\text { isolated from the stem } \\
\text { bark .( Sood } \text { et al. 2004): }\end{array}$ \\
\hline 25. & $\begin{array}{l}\text { Prunus } \\
\text { mira/Smoot } \\
\text { h pit peach/ } \\
\text { Aroo }\end{array}$ & $\begin{array}{l}\text { Up } \\
\text { to } \\
40 \\
00 \\
\text { m } \\
\text { (T) }\end{array}$ & $\begin{array}{l}\text { Aroo ki } \\
\text { darru or } \\
\text { tirul ki } \\
\text { darru }\end{array}$ & $(\mathrm{Ab})$ & (B) & $\mathrm{Fr}$ & $\begin{array}{l}\mathrm{Jg}, \text { Fr of } \\
\text { plant }\end{array}$ & Same as Prunus armeniaca & $\begin{array}{l}\text { Md:A massage of aroo } \\
\text { seed oil is recommended } \\
\text { for patients suffering from } \\
\text { disorders of joints. Fr } \\
\text { improves digestion, } \\
\text { respiration and gives a } \\
\text { sense of wellbeing }\end{array}$ \\
\hline 26. & $\begin{array}{l}\text { Prunus } \\
\text { persica } \\
\text { /peach/Aar } \\
u\end{array}$ & $\begin{array}{l}\text { Up } \\
\text { to2 } \\
50 \\
0 \mathrm{~m} \\
(\mathrm{~T})\end{array}$ & $\begin{array}{l}\text { Aaru ki } \\
\text { darru }\end{array}$ & $(\mathrm{Ab})$ & (B) & Fr & $\begin{array}{l}\text { Jg,Fr of } \\
\text { plant }\end{array}$ & Same as Prunus armeniaca & $\begin{array}{l}\text { Md:A pinch of dried Fl } \\
\text { taken as snuff to cure } \\
\text { headache. Infusion of Lf } \\
\text { \& Rt is useful for cold, } \\
\text { inflamed respiratory tract }\end{array}$ \\
\hline
\end{tabular}




\begin{tabular}{|c|c|c|c|c|c|c|c|c|c|}
\hline & & & & & & & & & $\begin{array}{l}\text { and body massages. Fr are } \\
\text { antiemetic, antiscorbutic, } \\
\text { ascaricide, demulcent, } \\
\text { diuretic, purgative, } \\
\text { stomachic. These are } \\
\text { ffective against eczema, } \\
\text { headache, scabies and } \\
\text { whooping cough. }\end{array}$ \\
\hline 27. & $\begin{array}{l}\text { Rosa } \\
\text { canina/Dog } \\
\text { rosel }\end{array}$ & $\begin{array}{l}\text { Up } \\
\text { to } \\
20 \\
00 \\
\text { m( } \\
\text { Sh) }\end{array}$ & $\begin{array}{l}\text { Van- } \\
\text { gulab ki } \\
\text { shraab }\end{array}$ & $(\mathrm{Ab})$ & $(\mathrm{W})$ & $\mathrm{Fr}, \mathrm{Fl}$ & $\begin{array}{l}\text { Jg, Fl \& } \\
\text { fr }\end{array}$ & Same as Bauhinia variegata & 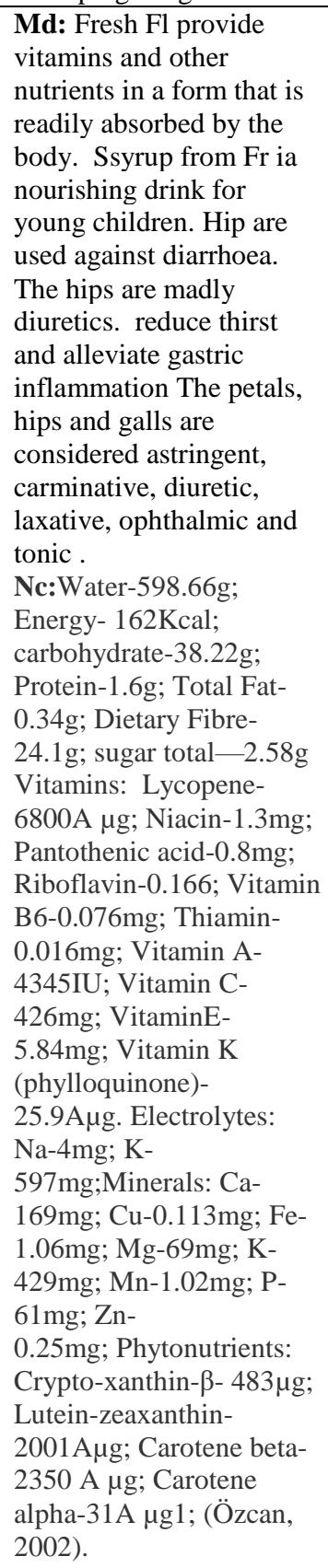 \\
\hline 28. & $\begin{array}{l}\text { Rubus } \\
\text { ellipticus/Y } \\
\text { ellow } \\
\text { Himalayan } \\
\text { rasberry/ } \\
\text { aakhe/Hire } \\
\text { ka kanta }\end{array}$ & $\begin{array}{l}\text { Up } \\
\text { to } \\
23 \\
00 \\
\text { m( } \\
\text { Sh) }\end{array}$ & $\begin{array}{l}\text { Aakhe ki } \\
\text { Desi } \\
\text { shraab/ } \\
\text { Darru }\end{array}$ & $(\mathrm{Ab})$ & $(\mathrm{W})$ & Rt & $\begin{array}{l}\text { Jg, Rt of } \\
\text { plant, } \\
\text { cinnamo } \\
\mathrm{n}, \\
\text { cardamo } \\
\text { m etc }\end{array}$ & Same as Berberis lycium & $\begin{array}{l}\text { Md: Decoction of Rt good } \\
\text { for cough, fever, } \\
\text { dysentery, malaria \& } \\
\text { stomach aliments Both } \\
\text { the Rt \& Ts are } \\
\text { considered to be a good } \\
\text { treatment for colic. Fr } \\
\text { juice is used in the } \\
\text { treatment of fever, colic, }\end{array}$ \\
\hline
\end{tabular}




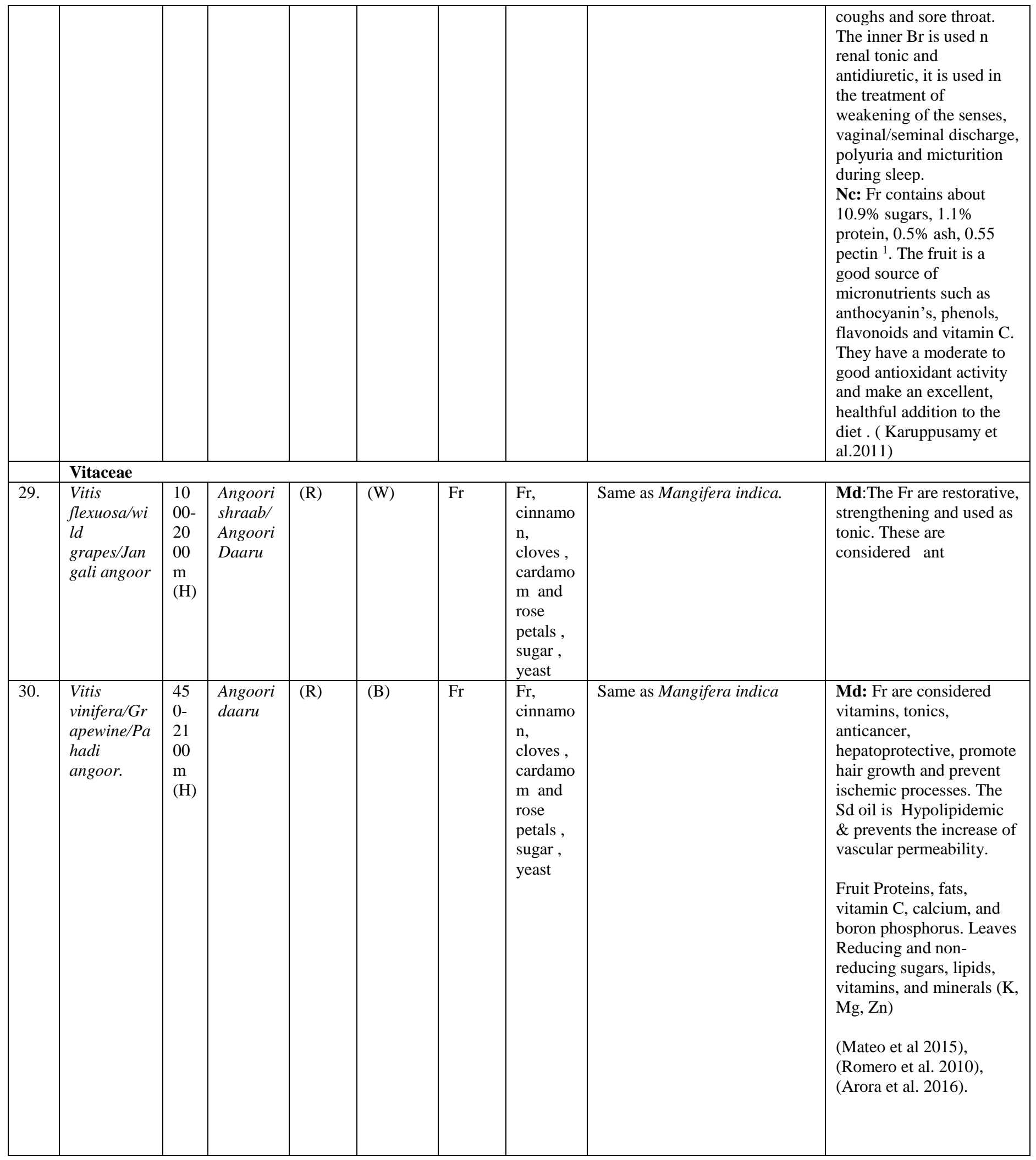




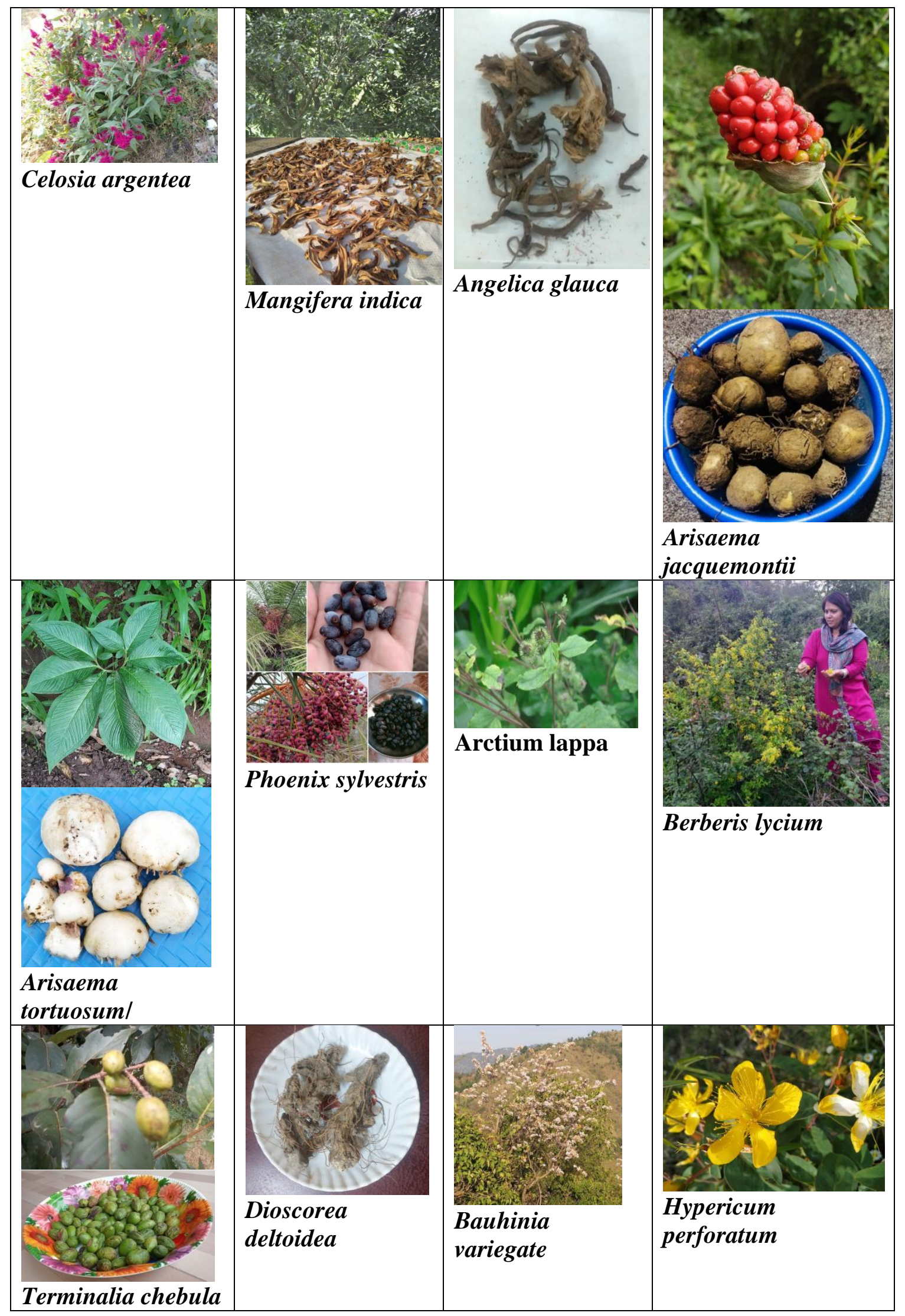

Copyright (C) 2020-2021, Journal of Scientific Research in Medical and Biological Sciences (JSRMBS), Under the license CC BY- 4.0 


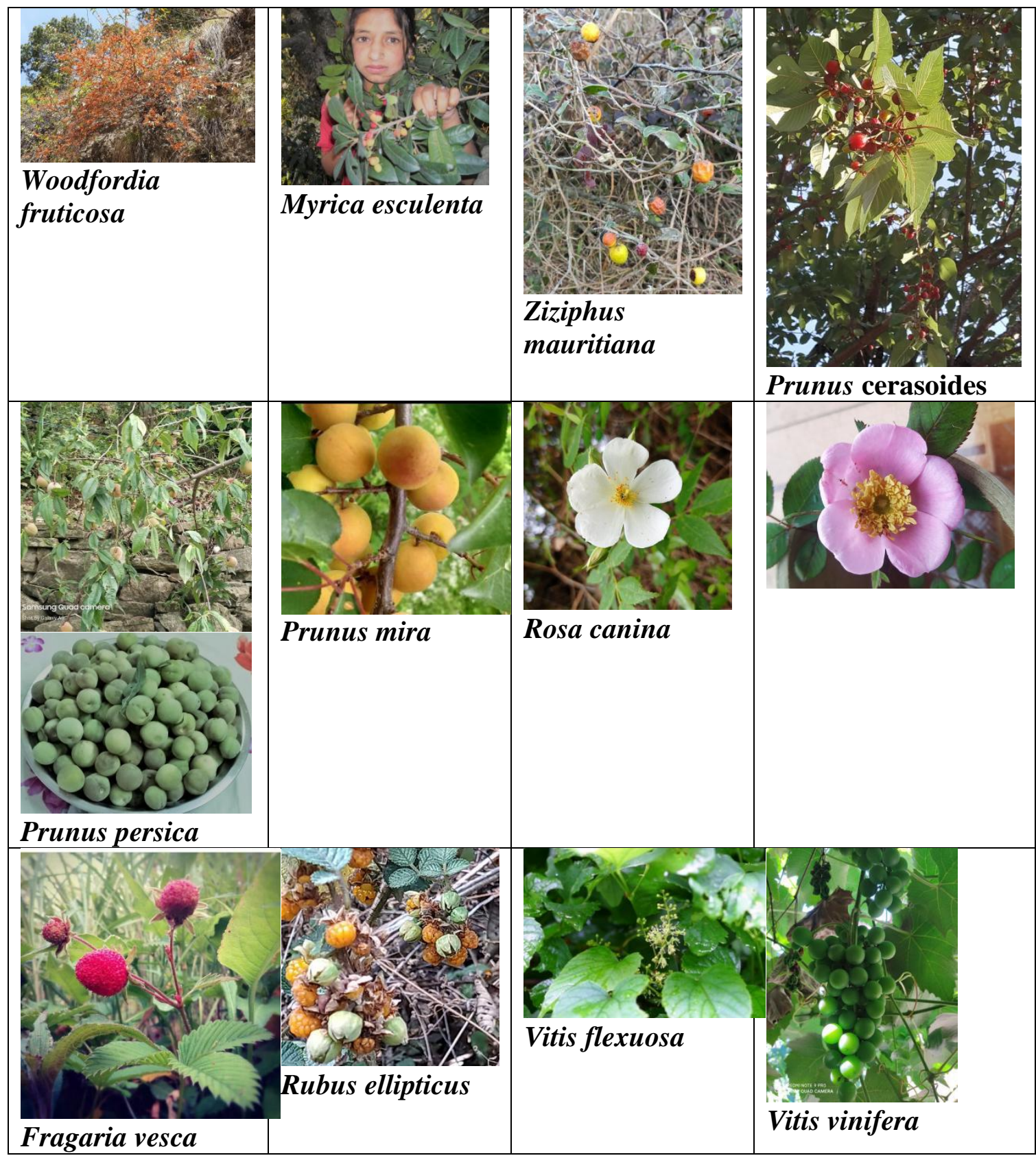

Figure 8: Images showing plants useful in making LADs.

Table 4: Herbal Ingredients used to make drinks

\begin{tabular}{|l|l|l|l|}
\hline Herbs & Family & Parts used & Life forms \\
\hline Achyranthus aspera & Amranthaceae & Lf & $\mathrm{H}$ \\
\hline Carissa spinarum & Apocynaceae & $\mathrm{fr}$ & $\mathrm{Sh}$ \\
\hline Centella asiatica & Apiaceae & $\mathrm{Wp}$ & $\mathrm{H}$ \\
\hline & & & \\
\hline Cinnamomum tamala & Lauraceae & $\mathrm{Lf}$ & $\mathrm{T}$ \\
\hline Citrus limon & Rutaceae & $\mathrm{Fr}$ & $\mathrm{T}$ \\
\hline Citrus sinensis & Rutaceae & $\mathrm{Fr}$ & $\mathrm{T}$ \\
\hline Cyclospermum leptophyllum & Apiaceae & $\mathrm{Lf}$ & $\mathrm{H}$ \\
\hline Dactylorhiza hatagirea & Orchidaceae & Rt & $\mathrm{H}$ \\
\hline Drymaria cordata & Caryophyllaceae & Young Lf & $\mathrm{H}$ \\
\hline Eclipta prostrata & Asteraceae & Young Lf & $\mathrm{H}$ \\
\hline Elettaria cardamomum & Zingiberaceae & Sd & $\mathrm{H}$ \\
\hline
\end{tabular}

94

Copyright @ 2020-2021, Journal of Scientific Research in Medical and Biological Sciences (JSRMBS), Under the license CC BY- 4.0 


\begin{tabular}{|l|l|l|l|}
\hline Euphorbia hirta & Euphorbiaceae & Lf & $\mathrm{H}$ \\
\hline Juglans regia & Juglandaceae & $\mathrm{Fr}$ & $\mathrm{T}$ \\
\hline Mentha longifolia & Lamiaceae & $\mathrm{Lf}$ & $\mathrm{H}$ \\
\hline Morchella esculenta & Agaricaceae & $\mathrm{Fr}$ (Mushroom) & $\mathrm{H}$ \\
\hline Moringa oleifera & Moringaceae & $\mathrm{Fr}$ & $\mathrm{T}$ \\
\hline Murraya koeingii & Rutaceae & $\mathrm{Lf}$ & $\mathrm{H}$ \\
\hline Phyllanthus emblica & Phyllanthaceae & $\mathrm{Fr}$ & $\mathrm{T}$ \\
\hline Rosa macrophylla & Rosaceae & $\mathrm{Fl}$ & $\mathrm{Sh}$ \\
\hline & & & \\
\hline Rubus niveus & Rosaceae & $\mathrm{Fr}$ & $\mathrm{Sh}$ \\
\hline Rumex acetosa & Polygonaceae & $\mathrm{Lf}$ & $\mathrm{H}$ \\
\hline Syzygium aromaticum & Myrtaceae & $\mathrm{Fl} \mathrm{buds}$ & $\mathrm{T}$ \\
\hline Selinum vaginatum & Apiaceae & $\mathrm{Tu}, \mathrm{Rt}, \mathrm{Lf}$ & $\mathrm{H}$ \\
\hline & & & \\
\hline Terminalia bellirica & Combertaceae & $\mathrm{Fr}$ & $\mathrm{T}$ \\
\hline Zanthoxylum armatum & Rutaceae & $\mathrm{Ts}$ & $\mathrm{Sh}$ \\
\hline
\end{tabular}

Abbreviations used: H=Herb; T=Tree; Sh=Shrub; St=Stem; Fr=Fruit;Bk=Bark; Rh= Rhizome; $S d=$ Seed; Bd=Buds Tu= tuber; Ap=Aerial Part; Lf=Leaf; Fl=Flower; $T s=$ Tender shoot.

\section{Types of LADs, their methods of their preparation \& Use Value Index}

LADs are categorized into 7 types as Distilled (5 spp),Un- distilled (3 spp), Aromatic (10 spp), Hard -cider (1sp), Lugadi (1sp), Whiskey (3spp), Medicinal(7spp) on the basis of their method of preparation and plant parts used. Details of each is listed in table 5. The relative importance of each of these LADs was assessed by calculating a general Use Value Index. UVI of aromatic drinks score highest (UVgeneral $=0.8$ ) followed by Whiskey $(0.56)$ and Lugadi (0.56). UVcurrent was maximum (0.4) for aromatic drinks followed by Distilled (0.36) and Hard -cider (0.3) effective value was again maximum for aromatic drinks $(0.4)$ followed by Distilled (0.36) and Hard -cider (0.3) (Table.5: Fig 9 \& 10).

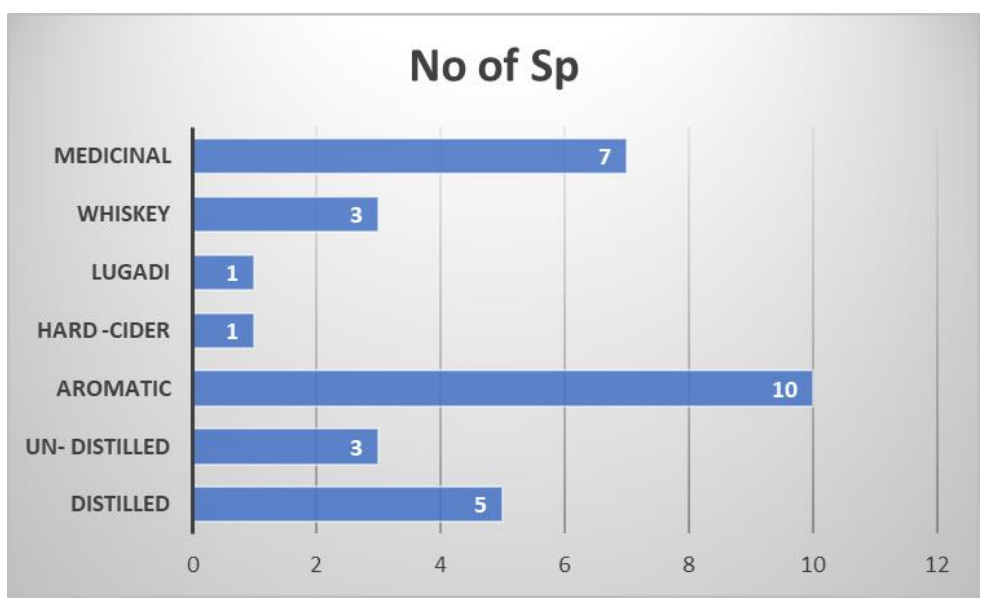

Figure 9: Types of local alcoholic drinks 


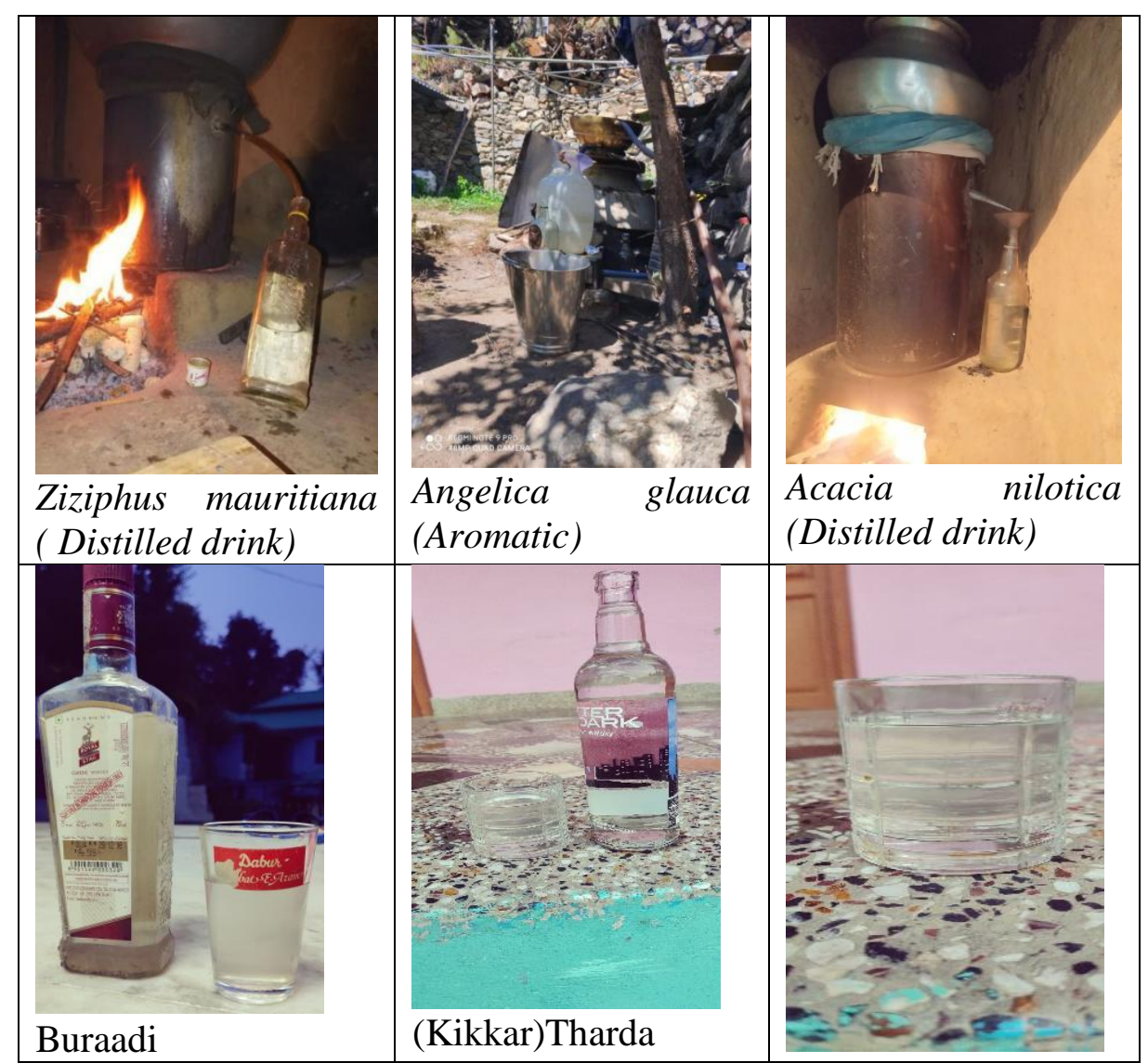

Figure 10: Images showing preparation of different types of local alcoholic drinks preparation

Table 5: Types of LADs

\begin{tabular}{|c|c|c|c|c|c|c|c|c|}
\hline \multirow[b]{2}{*}{ S.No. } & \multirow[b]{2}{*}{ Type } & \multirow[b]{2}{*}{$\begin{array}{l}\text { No. of } \\
\text { spp. }\end{array}$} & \multirow{2}{*}{$\begin{array}{c}\text { Citation } \\
\text { (Informants } \\
\text { mentioning the } \\
\text { plant) }\end{array}$} & \multicolumn{5}{|c|}{ Uv (used value) } \\
\hline & & & & General & Current & Past & Effective & Potential \\
\hline 1. & $\begin{array}{l}\text { Distilled } \\
\text { Phoenix sylvestris } \\
\text { Arctium lappa } \\
\text { Acacia nilotica } \\
\text { Woodfordia fruticosa } \\
\text { Ziziphus mauritiana }\end{array}$ & 5 & 11 & 0.36 & 0.36 & 0.18 & 0.36 & 0.18 \\
\hline 2. & $\begin{array}{l}\text { Un- distilled } \\
\text { Mangifera indica } \\
\text { Vitis flexuosa } \\
\text { Vitis vinifera }\end{array}$ & 3 & 8 & 0.26 & 0.26 & 0.00 & 0.13 & 0.13 \\
\hline 3. & $\begin{array}{l}\text { Aromatic } \\
\text { Celosia argentea } \\
\text { Angelica glauca } \\
\text { Bauhinia variegate } \\
\text { Hypericum perforatum } \\
\text { Prunus armeniaca } \\
\text { Prunus cerasoidess } \\
\text { Prunus persica } \\
\text { Prunus mira } \\
\text { Fragaria vesca } \\
\text { Rosa canina } \\
\end{array}$ & 10 & 12 & 0.8 & 0.4 & 0.2 & 0.4 & 0.2 \\
\hline 4. & $\begin{array}{l}\text { Hard -cider } \\
\text { Malus domestica }\end{array}$ & 1 & 9 & 0.3 & 0.3 & 0.15 & 0.3 & 0.00 \\
\hline 5. & $\begin{array}{l}\text { Lugadi } \\
\text { Oryza sativa }\end{array}$ & 1 & 15 & 0.5 & 0.25 & 0.00 & 0.25 & 0.00 \\
\hline 6. & Whiskey & 3 & 17 & 0.56 & 0.28 & 0.00 & 0.28 & 0.00 \\
\hline
\end{tabular}

Copyright @ 2020-2021, Journal of Scientific Research in Medical and Biological Sciences (JSRMBS), Under the license CC BY- 4.0 


\begin{tabular}{|c|c|c|c|c|c|c|c|c|}
\hline & $\begin{array}{l}\text { Hordeum vulgare } \\
\text { Paspalum scorbiculatum } \\
\text { Zea mays }\end{array}$ & & & & & & & \\
\hline 7. & $\begin{array}{l}\text { Medicinal } \\
\text { Arisaema jacquemontii } \\
\text { Arisaema tortuosum } \\
\text { Berberis lycium } \\
\text { Terminalia chebula } \\
\text { Dioscorea deltoidea } \\
\text { Myrica esculenta } \\
\text { Rubus ellipticus }\end{array}$ & 7 & 12 & 0.4 & 0.2 & 0.1 & 0.2 & 0.2 \\
\hline
\end{tabular}

\section{Use Value Index of plants species useful in LADs preparation:}

The relative importance of each species used in making LADs was assessed by calculating UVI. According to calculations the most important species was Prunus armeniaca, Dioscorea deltoidea \& Rosa canina (UVgeneral = 0.9 each) followed by Myrica esculenta (0.8), Oryza sativa (0.66), Phoenix sylvestris, Acacia nilotica \& Prunus cerasoides (0.6 each) Prunus persica (0.46) and Malus domestica (0.43). Species with the highest UVcurrent were Myrica esculenta, Arisaema jacquemontii \& Arisaema tortuosum (0.8 each ), Ziziphus mauritiana (0.52) and Bauhinia variegata (0.5). The highest UVeffective values were obtained by Arisaema jacquemontii \& Arisaema tortuosum (0. 8 each ), Celosia argentea (0.5), Phoenix sylvestris, Terminalia chebula \& Myrica esculenta (0.4 each) and Berberis lycium (0.32).

Table 6: Used value (Uv) of plants species used in LADs preparation

\begin{tabular}{|c|c|c|c|c|c|c|c|c|}
\hline \multirow[b]{2}{*}{$\begin{array}{c}\text { S.n } \\
\text { o }\end{array}$} & \multirow[b]{2}{*}{ Botanica name } & \multirow{2}{*}{$\begin{array}{c}\text { Informa } \\
\text { nts } \\
\text { mention } \\
\text { ing the } \\
\text { plants }\end{array}$} & \multirow[b]{2}{*}{$\begin{array}{c}\text { citati } \\
\text { on }\end{array}$} & \multicolumn{5}{|c|}{ Used value } \\
\hline & & & & $\begin{array}{c}\text { Gener } \\
\text { al }\end{array}$ & $\underset{\text { nt }}{\text { Curre }}$ & Past & $\begin{array}{l}\text { Effecti } \\
\text { ve }\end{array}$ & $\begin{array}{c}\text { Potenti } \\
\text { al }\end{array}$ \\
\hline 1. & Celosia argentea & 3 & 3 & 0.1 & 0.1 & 0.0 & 0.5 & 0.0 \\
\hline 2. & Mangifera indica & 8 & 9 & 0.39 & 0.26 & 0.13 & 0.26 & 0.13 \\
\hline 3. & Angelica glauca & 4 & 4 & 0.13 & 0.00 & 0.7 & 0.00 & 0.13 \\
\hline 4. & Arisaema jacquemontii & 4 & 5 & 0.16 & 0.8 & 0.00 & 0.8 & 0.00 \\
\hline 5. & Arisaema tortuosum & 4 & 5 & 0.16 & 0.8 & 0.0 & 0.8 & 0.0 \\
\hline 6. & Phoenix sylvestris & 6 & 5 & 0.6 & 0.2 & 0.2 & 0.4 & 0.4 \\
\hline 7. & Arctium lappa & 2 & 2 & 0.00 & 0.00 & 0.033 & 0.00 & 0.033 \\
\hline 8. & Terminalia chebula & 12 & 12 & 0.16 & 0.4 & 0.8 & 0.4 & 0.8 \\
\hline 9. & Dioscorea deltoidea & 9 & 9 & 0.9 & 0.3 & 0.0 & 0.1 & 0.6 \\
\hline 10. & Acacia nilotica & 6 & 5 & 0.6 & 0.2 & 0.1 & 0.2 & 0.4 \\
\hline 11. & Bauhinia variegata & 15 & 4 & 0.15 & 0.5 & 0.2 & 0.1 & 0.5 \\
\hline 12. & Hypericum perforatum & 12 & 11 & 0.8 & 0.4 & 0.2 & 0.1 & 0.4 \\
\hline 13. & Woodfordia fruticosa & 4 & 3 & 0.26 & 0.13 & 0.7 & 0.13 & 0.7 \\
\hline 14. & Myrica esculenta & 5 & 8 & 0.16 & 0.8 & 0.0 & 0.4 & 0.0 \\
\hline 15. & Hordeum vulgare & 6 & 7 & 0.4 & 0.2 & 0.0 & 0.2 & 0.4 \\
\hline 16. & Oryza sativa & 10 & 11 & 0.66 & 0.33 & 0.16 & 0.32 & 0.33 \\
\hline 17. & Paspalum scorbiculatum & 12 & 10 & 0.16 & 0.4 & 0.2 & 0.2 & 0.4 \\
\hline 18. & Zea mays & 10 & 9 & 0.33 & 0.00 & 0.00 & 0.00 & 0.33 \\
\hline 19. & Ziziphus mauritiana & 16 & 15 & 0.00 & 0.52 & 0.265 & 0.00 & 0.265 \\
\hline 20. & Malus domestica & 13 & 11 & 0.43 & 0.00 & 0.00 & 0.00 & 0.23 \\
\hline 21. & Prunus armeniaca & 9 & 8 & 0.9 & 0.3 & 0.1 & 0.3 & 0.3 \\
\hline 22. & Prunus cerasoides & 6 & 6 & 0.6 & 0.2 & 0.0 & 0.2 & 0.0 \\
\hline
\end{tabular}

Copyright (C) 2020-2021, Journal of Scientific Research in Medical and Biological Sciences (JSRMBS), Under the license CC BY- 4.0 


\begin{tabular}{|l|l|l|l|l|l|l|l|l|}
\hline 23. & Prunus persica & 7 & 8 & 0.46 & 0.23 & 0.11 & 0.0 & 0.11 \\
\hline 24. & Rosa canina & 9 & 9 & 0.9 & 0.0 & 0.0 & 0.0 & 0.0 \\
\hline 25. & Berberis lycium & 11 & 10 & 0.36 & 0.34 & 0.18 & 0.34 & 0.18 \\
\hline 26. & Rubus ellipticus & 3 & 3 & 0.4 & 0.2 & 0.0 & 0.2 & 0.0 \\
\hline 27. & Prunus mira & 4 & 6 & 0.13 & 0.0 & 0.7 & 0.0 & 0.7 \\
\hline 28. & Fragaria vesca & 2 & 2 & 0.066 & 0.00 & 0.00 & 0.00 & 0.33 \\
\hline 29. & Vitis flexuosa & 4 & 5 & 0.133 & 0.133 & 0.00 & 0.133 & 0.00 \\
\hline 30. & Vitis vinifera & 2 & 3 & 0.00 & 0.033 & 0.033 & 0.00 & 0.00 \\
\hline
\end{tabular}

\section{DMR value and indigenous uses of plants useful in making of LADs}

DMR value was calculated for each species to know the other indigenous uses of each plant species like fuel, fodder, food, medicine, construction of agricultural tools and religious uses beside their usefulness in making of LADs. DMR value was found highest for Terminalia chebula (26) followed by Bauhinia variegata (25) and Prunus cerasoides (24) showing their significant multiple uses for the local people. All the species were edible and used as food beside their use in making of LADs (DMR score 122) followed by their medicinal uses with DMR score 119 and their use to feed cattle with DMR score 81 for fodder

Table 7: DMR score for 30 plants species with additional uses besides their uses in LADs

\begin{tabular}{|l|c|c|c|c|c|c|c|}
\hline \multicolumn{1}{|c|}{ Botanical name } & $\begin{array}{c}\text { Agricult } \\
\text { ural } \\
\text { Tools }\end{array}$ & Fodder & Food & Fuel & Medicinal & Religious & Rank \\
\hline Celosia argentea & 0 & 2 & 4 & 0 & 5 & 3 & 14 \\
\hline Mangifera indica & 4 & 2 & 5 & 2 & 5 & 5 & 23 \\
\hline Angelica glauca & 0 & 3 & 4 & 0 & 5 & 0 & 12 \\
\hline $\begin{array}{l}\text { Arisaema } \\
\text { jacquemontii }\end{array}$ & 0 & 1 & 1 & 0 & 4 & 0 & 6 \\
\hline Arisaema tortuosum & 0 & 1 & 1 & 0 & 4 & 0 & 6 \\
\hline Phoenix sylvestris & 4 & 4 & 4 & 0 & 4 & 5 & 21 \\
\hline Arctium lappa & 0 & 3 & 3 & 0 & 5 & 0 & 11 \\
\hline Terminalia chebula & 4 & 3 & 5 & 4 & 5 & 5 & 26 \\
\hline Dioscorea deltoidea & 0 & 4 & 5 & 0 & 5 & 0 & 14 \\
\hline Acacia nilotica & 2 & 4 & 4 & 3 & 4 & 0 & 17 \\
\hline Bauhinia variegata & 4 & 5 & 5 & 5 & 4 & 2 & 25 \\
\hline $\begin{array}{l}\text { Hypericum } \\
\text { perforatum }\end{array}$ & 0 & 3 & 2 & 0 & 4 & 2 & 11 \\
\hline Woodfordia fruticosa & 1 & 3 & 2 & 1 & 4 & 1 & 12 \\
\hline Myrica esculenta & 3 & 3 & 5 & 3 & 3 & 0 & 17 \\
\hline Hordeum vulgare & 0 & 4 & 5 & 0 & 3 & 5 & 17 \\
\hline Oryza sativa & 0 & 5 & 5 & 0 & 3 & 5 & 18 \\
\hline $\begin{array}{l}\text { Paspalum } \\
\text { scorbiculatum }\end{array}$ & 0 & 3 & 5 & 0 & 4 & 0 & 12 \\
\hline Zea mays & 0 & 5 & 5 & 2 & 3 & 5 & 20 \\
\hline Ziziphus mauritiana & 1 & 2 & 5 & 1 & 4 & 0 & 13 \\
\hline Malus domestica & 3 & 2 & 5 & 3 & 3 & 3 & 19 \\
\hline Prunus armeniaca & 4 & 1 & 5 & 4 & 4 & 2 & 20 \\
\hline Prunus cerasoides & 4 & 5 & 2 & 4 & 4 & 5 & 24 \\
\hline Prunus persica & 4 & 1 & 5 & 4 & 4 & 4 & 22 \\
\hline Rosa canina & 0 & 1 & 3 & 0 & 4 & 4 & 12 \\
\hline
\end{tabular}




\begin{tabular}{|l|c|c|c|c|c|c|c|} 
Berberis lycium & 0 & 0 & 3 & 1 & 4 & 0 & 8 \\
\hline Rubus ellipticus & 0 & 1 & 5 & 0 & 3 & 0 & 9 \\
\hline Prunus mira & 4 & 1 & 5 & 4 & 4 & 4 & 22 \\
\hline Fragaria vesca & 0 & 1 & 4 & 0 & 3 & 0 & 8 \\
\hline Vitis flexuosa & 0 & 3 & 5 & 0 & 4 & 0 & 12 \\
\hline Vitis vinifera & 0 & 3 & 5 & 0 & 4 & 0 & 12 \\
\hline Rank & 42 & 81 & 122 & 41 & 119 & 60 & \\
\hline
\end{tabular}

Criteria used for data collection and analysis $(0=$ not used, $1=$ least used, $2=$ less, $3=$ good, $4=$ very good,5=best)

\section{Medicinal \& Nutraceutical effects of LADs}

All the plants listed in this study are known to have some medicinal value and can cure a wide range of ailments (listed in Table.4), but with passage of time their use has been declined progressively and at present very few plants were actually being practiced for their medicinal purposes. Discussion with informants reveals a significant gap between documented and actual medicinal uses. Only 7 species out of 30 are chiefly used for their medicinal values in preparation of LADs. Maximum plants used in making LADs are rich in nutraceutical value (Table.4) and contribute to boost immunity and health with justified use of LADs.

\section{Conclusion}

Drinking alcohol was an occasional activity shared by people within the communities and their consumption chiefly associated with festivals, special occasions and other social gatherings In many cultures. (WHO, 2014). It was found an essential activity in almost all the communities of study area during marriages, festivals and other rituals sowing religious as well as socio-economic importance of alcoholic drinks, so these can have health or therapeutic benefits to communities at large if made of medicinal plants or by including herbal ingredients. But at present the consumption of the LADs has been gradually coming down and largely replaced by commercially available alcoholic drinks, because of advancement in literacy, urbanization, modernization, enforcement of the excise law and economic pressure.

Thus, a sharp decline has been noticed in the preparation and consumption of LADs. As a result their use and selling is in restricted manner and that too is mainly connected with festivals and ceremonies. Similar findings were shown by work done by other researchers also (Das A. 2016; Łuczaj et al. 2019). Still LADs occupy an important place in the traditional culture and social life of local people in this region of the world, specially in the life of the people residing in temperate region. Retrieving local knowledge of LADs could be regarded as a means not only of contributing to the conservation of local traditional knowledge, but also of reevaluating a possible source of socio-economic endogenous rural development and tourism growth. As large-scale production of LADs for commercial use is restricted in study area, so people usually prepare these drinks for local personal use or trade illegally to people of nearby villages. As local food, beverages, culture and scenic beauty of any tourist destination is the focal point in the travel plans of holiday makers while they choose any tourist destination (Devi $\&$ Sen, 2021). So, LADs specially aromatic \& medicinal drinks are main tourism attraction in some of hilly tourist destinations of the study area and help in attracting visitor's interested in 
local traditional food and beverages which are made with local natural resources. Thus LADs also play important role in boosting health, immunity \& rural tourism. Medicinal and nutraceutical properties of herbal ingredients listed in this study further enhance the value and uniqueness of LADs prepared in the study area. So, LADs can be commercialized after proper lab testing.

As among 30 listed plant species useful in LADs preparation 18 species are wild, 9 of which are rare and 3 are already listed in endangered category of IUCN list of Threatened Species. So it is recommended to sustainably harvest essential plant parts of these plants like fruits, flowers or roots which are important means of future regeneration and propagation. These plants can be further cultivated or domesticated on large scale by mass awareness for ensuring their conservation and sustainable utilization.

\section{Limitation of Study}

The list of plants useful in making of LADs found in Western Himalayas is yet to be completed.

\section{Conflict of Interest}

None

\section{Funding}

DEST Himachal Pradesh

\section{Acknowledgments}

The author is highly indebted to the inhabitants of Himachal Pradesh for providing valuable information about wild food plants locally used as a tonic and helps to cure cold, fever, cough and bronchitis Sincere thanks also go to Dr S.S. Samant, Dr. Chiranjit Parmar, Dr, Sanjeet Singh, Dr. Neha Sen, Vijay Sen, Bhavana Bhardwaj, Manoj Kumar, Archana, Chetna Thakur. official of district statistical department, botany department of Sardar Vallabh bhai Cluster University Mandi for their encouragement, support and cooperation. The author is also highly thankful to DEST Himachal Pradesh for providing funds to carry on this research.

\section{References:}

Ali S, Masud T, Abbasi KS.(2011); Physico-chemical characteristics of apricot (Prunus armeniaca L.) grown in Northern Areas of Pakistan. Scientia Horticulture, 130(2):386-392.

Anonymous (1969) : The Wealth of India, Raw Materials,Vol VIII, Publications and Information Directorate, CSIR, New Delhi,

Anonymous, (1988), Wealth of India:Raw Materials, Vol II, CSIR, New Delhi.

Arora, P.; Ansari, S.H.; Najmi, A.K.; Anjum, V.; Ahmad, S. Investigation of anti-asthmatic potential of dried fruits of Vitis vinifera L. in animal model of bronchial asthma. Allergy, Asthma \& Clinical Immunology 2016, 12, 42, https://doi.org/10.1186/s13223-016-0145-x

Austin GA. (1985).Alcohol in Western Society from Antiquity to 1800. A chronological history. Santa Barbara: ABC Clio. 
Boyer, J.; Liu, R.H. (2004): Apple phytochemicals and their health benefits. Nutr. J.3, 5-20.

Chandel G, Meena RK, (2014)Dubey M, Kumar M. Nutritional properties of minor millets: neglected cereals with potentials to combat malnutrition. Current Science. ; 107(7):1109-1111.et al

Chowdhery HJ, Wadhwa BM.(1984): "Flora of Himachal Pradesh", Botanical Survey of India, Culcutta. Vol. 1-3.

Collett H.(1902) "Flora Simlensis", Bishen Singh Mahendra Pal Singh, Dehradun.

Das, A. (2016). Medicinal plants used traditionally for the preparation of rice beer by the Tiwa tribe of Morigaon district of Assam, India. Int. J. Curr. Res, 8(11), 40940-40943.

De Cleene M, Lejeune MC. Compendium of symbolic and ritual plants in Europe, vol 1 (trees and shrubs), vol 2 (herbs). Ghent: Mens \& Cultuur Uitgevers; 2003.

Deka D, Sarma GC.( 2010) Traditionally used herbs in the preparation of rice-beer by the Rabha tribe of Goalpara district, Assam. Indian J Tradit Know.;9: 459-62.

Devi T \& Sen V. (2020): "Role of Wild Food Plants in Culinary Tourism Development of District Mandi Pradesh," International Journal of Scientific Research in .Sciences, Vol.7, Issue.6, pp.14

Duke. J. A.\& Ayensu. E. S. (1985); Medicinal Plants of China reference Publications, Inc. ISBN 0-917256-20-4

Egea, T., Signorini, M. A., Bruschi, P., Rivera, D., Obón, C., Alcaraz, F., \& Palazón, J. A. (2015). Spirits and liqueurs in European traditional medicine: Their history and ethnobotany in Tuscany and Bologna (Italy). Journal of ethnopharmacology, 175, 241-255.

Egea T, Signorini MA, Ongaro L, Rivera D, de Castro CO, Bruschi P. (2016); Traditional alcoholic beverages and their value in the local culture of the Alta Valle del Reno, a mountain borderland between Tuscany and EmiliaRomagna (Italy). J Ethnobiol Ethnomed. 12(1):27

Gulfraz M, Arshad M, Nayyer N, Kanwal N, Nisar U (2004). Investigation for bioactive compounds of Berberis lyceum Royle and Justicia adhatoda L. Ethnobot. Leaflets 1:51-62.

Gurrieri F, Audergon JM, Albagnac G, Reich M.(2001);Soluble sugars and carboxylic acids in ripe apricot fruit as parameters for distinguishing different cultivars. Euphytica. 117:183-189.

Karuppusamy, S., Muthuraja, G., \& Rajasekaran, K. M. (2011). Antioxidant activity of selected lesser known edible fruits from Western Ghats of India.

Kumar, D., \& Jhariya, N. A. (2013). Nutritional, medicinal and economical importance of corn: A mini review. Research Journal of Pharmaceutical Sciences, 2, 7-8.

Leccese A, Bartolini S, Viti R. (2007); Total antioxidant capacity and phenolics content in apricot fruits International Journal of Fruit Science. 7(2): 3-16.

Lichou, J, Jay M, Vaysse P,(2003) Lespinasse N. Reconnaitre les variétésd, abricots. Editions Ctifl, France, 17-29

Luczaj et al. Journal of Ethnobiology and Ethnomedicine (2019) 15:51 https://doi.org/10.1186/s13002-019-0332-1 
Madej T, Pirożnikow E, Dumanowski J, Łuczaj Ł(2014). Juniper beer in Poland: the story of the revival of a traditional beverage. J Ethnobiol. ;34(1):84-103

Das M \& Kau S.(2016) "Status of Barley as a Dietary Component for Human”. Journal of Food and Dairy Technology $4.1: 25-28$.

Morton J.(1987) Indian Jujube. http://www.hort. purdue.edu/newcrop/morton/ indian_jujube. html (assessed on 11.07.2020),

Mateo, J.J.; Maicas, S. Valorization of winery and oil mill wastes by microbial technologies. Food Res. Int. 2015, 73, 13-25, https://doi.org//10.1016/j.foodres.2015.03.007. 29.

Munzuroglu O, Karatas F, Geckil H. (2003); The vitamin and selenium contents of apricot fruit of different varieties cultivated in different geographical regions. Food Chemistry. 83:205-212.

Muragod PP, Muruli NV, Padeppagol S, Kattimani A.(2019); Physico-Chemical Properties and Nutritional Factors of Kodo Millet. Int. J Pure App. Biosci. 7(1):117-123.

Nour, V.; Trandafir, I.; Ionica, M.E. (2010):Compositional characteristics of fruits of several apple (Malus domestica Borkh.) cultivars. Not. Bot. Hort. Agrobot. Cluj , 38, 228-233

(Nutrional Evaluation, Antimicrobial Activity And Phyto chemical Screening Of Wild Edible Fruit of Myrica nagi pulp)

Özcan, M. (2002). Nutrient composition of rose (Rosa canina L.) seed and oils. Journal of Medicinal Food, 5(3), 137-140

Pareek S, Dhaka RS.(2008);Association analysis for quality attributes in ber. Ind. J. Arid Hort. 3:77-80.

Pareek S, Kitinoja L, Kaushik RA, Paliwal R. (2009);Postharvest physiology and storage of ber. Stew. Posthar. Rev. 5(5):1-10.

Parmar. C. and Kaushal. M.K. (1982) Wild Fruits of the Sub-Himalayan Region. Kalyani Publishers. New Delhi. possible. Chem., 67(2): 209-212.

Purohit SS, Sharma AK, Prajapati ND, Kumar T. (2009); A handbook of medicinal plants: a complete source book. 2:352-3.

Richardson, A.T.; Cho, J.; McGhie, T.K.; Larsen, D.S.; Schaffer, R.J.; Espley, R.V.; Perry, N.B.( 2020): Discovery of a stable vitamin $C$ glycoside in crab apples (Malus sylvestris). Phytochemistry, 173, 112297.

Romero, I.; García-Escudero, E.; Martín, I. Effects of Leaf Position on Blade and Petiole Mineral Nutrient Concentration of Tempranillo Grapevine $(<\mathrm{em}>$ Vitis vinifera $</ \mathrm{em}>$ L.). American Journal of Enology and Viticulture 2010, 61, 544, https://doi.org/10.5344/ajev.2010.09091.

Rossato SVC, Leitao-Filho H, Begossi A(1999). Ethnobotany of Caiçaras of the Atlantic forest coast (Brazil). Econ Bot. ;53:387-95.

Roy B., Kala C. P., Farooquee NA.(2004); Majila B.S.Indigenous Fermented Food and Beverages: A Potential for Economic Development of the High Altitude Societies in Uttaranchal. Journal of Human Ecology. 15(1): 45-49.

Saikia B., Tag H; Das A.K. (2007);Ethno botany of foods and beverages among the rural farmers of Tai Ahom of North Lakhimpur District,Asom. Indian journal of traditional knowledge. 6(1):126-132. 
Sen, T. D. (2021). The Role of Wild Food Plants of Himachal Pradesh in Boosting Immunity to Combat COVID-19. Journal of Scientific Research in Medical and Biological Sciences, 2(2), 23-62. https://doi.org/10.47631/jsrmbs.v2i2.238

Signorini MA, Piredda M, Bruschi P. (2009): Plants and traditional knowledge: An ethnobotanical investigation on Monte Ortobene (Nuoro, Sardinia). J Ethnobiol Ethnomed. ;5:6.

Singh PB, Singh (1918) "Flora of the Mandi District Himachal Pradesh North West Himalaya". Bishen Singh Mahendra Pal Singh, Dehradun. .

Śliwińska M, Wiśniewska P, Dymerski T, Wardencki W, Namieśnik J. (2017);1 Authenticity assessment of the "Onisiówka" Nalewka liqueurs using twodimensional gas chromatography and sensory evaluation. Food Anal Meth. 0(6):1709-20.

Sood SK, Thakur S. (2004): Ethnobotany of Rewalsar Himalaya. Deep Publications.

Sotelo, A., V. Sousa and I. Montalvo, (1990). ChemicalComposition of Different Fractions of 12 Mexican

Tanveer M, Habib-Ur-Rehman, Mesaik M, Choudhary M.( 2014); Immunomodulatory, antileishmanial and phytotoxicity of Arisaema jacquemontii Blume plant extracts. Archives of Applied Science Research. 6:12-17.

Tardío J, Pardo-de-Santayana M, Morales R. Ethnobotanical review of wild edible plants in Spain. Bot J Linn Soc. 2006;152(1):27-71.

Thakur, ST. (2021);An Illustrated Guide To Some Wild growing Food Plants of The Sub Himalayan Region. Narayan Publication.

Tharanathan, R. N., H. M. Yashoda, and T. N. Prabha. (2006 "Mango (Mangifera indica L.),"The king of fruits"-An overview." Food Reviews International 22.2): 95-123

USDA. (2010): Department of Agriculture Nutrient Data Laboratory 2010: USDA database for the oxygen radical absorbance capacity (ORAC) of selected foods, Release. Varieties of Rice Obtained During Milling. Cereal

Wanasundera, J. P. D., and G. Ravindran.(1994)"Nutritional assessment of yam (Dioscorea alata) tubers." Plant Foods for Human Nutrition 46.1 : 33-39.

WHO (2014). Global status report on alcohol and health. 